\title{
Consecutive up-down patterns in up-down permutations
}

\author{
Jeffrey B. Remmel \\ Department of Mathematics \\ University of California, San Diego \\ La Jolla, CA 92093-0112, U.S.A. \\ jremmel@ucsd.edu
}

Submitted: Mar 18, 2013; Accepted: Jun 21, 2014; Published: Jul 3, 2014

Mathematics Subject Classifications: 05A15, 05E05

\begin{abstract}
In this paper, we study the distribution of the number of consecutive pattern matches of the five up-down permutations of length four, 1324, 2314, 2413, 1432, and 3412 , in the set of up-down permutations. We show that for any such $\tau$, the generating function for the distribution of the number of consecutive pattern matches of $\tau$ in the set of up-down permutations can be expressed in terms of what we call the generalized maximum packing polynomials of $\tau$. We then provide some systematic methods to compute the generalized maximum packing polynomials for such $\tau$.
\end{abstract}

\section{Introduction}

If $\sigma=\sigma_{1} \ldots \sigma_{n}$ is a permutation in the symmetric group $S_{n}$, then we let

$$
\operatorname{Des}(\sigma)=\left\{i: \sigma_{i}>\sigma_{i+1}\right\} \text { and } \operatorname{Ris}(\sigma)=\left\{i: \sigma_{i}<\sigma_{i+1}\right\}
$$

Let $\mathbb{N}=\{0,1, \ldots$,$\} denote the natural numbers, \mathbb{P}=\{1,2, \ldots\}$ denote the set of positive integers, $\mathbb{E}=\{0,2,4, \ldots\}$ denote the set of even numbers in $\mathbb{N}$, and $[n]=\{1,2, \ldots, n\}$ for $n \in \mathbb{P}$. We say that $\sigma=\sigma_{1} \ldots \sigma_{n}$ is an up-down permutation if either $n=1$ or $n>1$ and $\operatorname{Des}(\sigma)=[n-1] \cap \mathbb{E}$. That is, we have

$$
\sigma_{1}<\sigma_{2}>\sigma_{3}<\sigma_{4} \ldots
$$

We let $\mathcal{A}_{n}$ denote the set of up-down permutations in $S_{n}$.

Given a sequence $\sigma=\sigma_{1} \ldots \sigma_{n}$ of distinct integers, let $\operatorname{red}(\sigma)$ be the permutation found by replacing the $i$ th smallest integer that appears in $\sigma$ by $i$. For example, if $\sigma=2754$, then $\operatorname{red}(\sigma)=1432$. Given a permutation $\tau=\tau_{1} \ldots \tau_{j} \in S_{j}$ and a permutation 
$\sigma=\sigma_{1} \ldots \sigma_{n} \in S_{n}$, we say that $\tau$ occurs in $\sigma$ if there are $1 \leqslant i_{1}<\ldots<i_{j} \leqslant n$ such that $\operatorname{red}\left(\sigma_{i_{1}} \ldots \sigma_{i_{j}}\right)=\tau$ and we say that $\sigma$ has a $\tau$-match starting at position $i$ in $\sigma$ if $\operatorname{red}\left(\sigma_{i} \sigma_{i+1} \ldots \sigma_{i+j-1}\right)=\tau$. We say that $\sigma$ avoids $\tau$ if there are no occurrences of $\tau$ in $\sigma$. We let $\tau$-mch $(\sigma)$ denote the number of $\tau$-matches in $\sigma$. We let $A_{n, \tau}(x)=\sum_{\sigma \in \mathcal{A}_{n}} x^{\tau-\operatorname{mch}(\sigma)}$.

These definitions are easily extended to sets of permutations $\Upsilon \subseteq S_{j}$. For example, we say that $\sigma$ has a $\Upsilon$-match starting at position $i$ in $\sigma$ if $\operatorname{red}\left(\sigma_{i} \sigma_{i+1} \ldots \sigma_{i+j-1}\right) \in \Upsilon$. We let $\Upsilon$-mch $(\sigma)$ denote the number of $\Upsilon$-matches in $\sigma$. We let $\mathcal{A}_{n, \Upsilon}(x)=\sum_{\sigma \in \mathcal{A}_{n}} x^{\Upsilon \text {-mch }(\sigma)}$.

There have been several papers that have studied the number of up-down permutations $\sigma \in \mathcal{A}_{n}$ which avoid a given pattern. For example, Mansour [19] and Deutsch and Reifegerste (see [27, Problem $h^{7}$ ] or [14]) showed that for any $\tau \in S_{3}$, the number of up-down permutations $\sigma \in \mathcal{A}_{n}$ which avoid $\tau$ is always a Catalan number. For example, the number of up-down permutations $\sigma \in \mathcal{A}_{n}$ that avoid $132(231)$ is $C_{\lfloor n / 2\rfloor}$. In [18], it was shown that the number of $\sigma \in \mathcal{A}_{2 n}$ that avoids 1234 or 2143 is $\frac{2(3 n) !}{n !(n+1) !(n+2) !}$. There has been somewhat less work on the distribution of $\tau$-matches in up-down permutations. Carlitz [5] found the generating function for the number of rises in the peaks of the updown permutations where a rise in the peaks of an up-down permutation is just 213-match in $\sigma$.

The main goal of this paper is to study the generating functions

$$
A_{\tau}(t, x)=1+\sum_{n \geqslant 1} \frac{t^{2 n}}{(2 n) !} \sum_{\sigma \in \mathcal{A}_{2 n}} x^{\tau-\operatorname{mch}(\sigma)}
$$

and

$$
B_{\tau}(t, x)=\sum_{n \geqslant 1} \frac{t^{2 n-1}}{(2 n-1) !} \sum_{\sigma \in \mathcal{A}_{2 n-1}} x^{\tau-\operatorname{mch}(\sigma)}
$$

in the case where $\tau \in \mathcal{A}_{4}$. Note that there are 5 permutations in $\mathcal{A}_{4}$, namely,

$$
\tau^{(1)}=1324, \tau^{(2)}=2314, \tau^{(3)}=2413, \tau^{(4)}=1423, \text { and } \tau^{(5)}=3412 .
$$

In fact, the main ideas of this paper can be extended to study the distributions of $\tau$ matches in the set of up-down permutations where $\tau$ is a natural analogue of a minimal overlapping permutation as studied by Duane and Remmel [8]. This work appears in Duane's thesis [7]. We have chosen to focus on the five up-down permutations of length four because the arguments are simpler and the formulas are more tractable than in the general case considered in Duane's thesis. However such an extension will be the subject of a forthcoming paper.

Let $\tau \in \mathcal{A}_{4}$. If $\sigma \in \mathcal{A}_{n}$ where $n \geqslant 4$, then $\tau$-matches can only start at odd positions. If $\sigma \in \mathcal{A}_{2 n}$ and $\tau$-mch $(\sigma)=n-1$, then we say that $\sigma$ is a maximum packing for $\tau$. Thus if $\sigma \in \mathcal{A}_{2 n}$ is a maximum packing for $\tau$, then $\sigma$ has $\tau$-matches starting at positions $1,3, \ldots, 2 n-3$. We let $\mathcal{M P}_{2 n, \tau}$ denote the set of maximum packings for $\tau$ in $\mathcal{A}_{2 n}$ and we let $\mathrm{mp}_{2 n, \tau}=\left|\mathcal{M P}_{2 n, \tau}\right|$. We shall see that it follows from results of Harmse and Remmel [13] that

$$
\mathrm{mp}_{2 n, \tau^{(1)}}=\operatorname{mp}_{2 n, \tau^{(3)}}=C_{n-1} \text { and }
$$




$$
\mathrm{mp}_{2 n, \tau^{(2)}}=\mathrm{mp}_{2 n, \tau^{(4)}}=\operatorname{mp}_{2 n, \tau^{(5)}}=1,
$$

where $C_{n}=\frac{1}{n+1}\left(\begin{array}{c}2 n \\ n\end{array}\right)$ is the $n$th Catalan number.

Our main theorem will show that for each $i \in[5]$, the generating functions $A_{\tau^{(i)}}(t, x)$ and $B_{\tau^{(i)}}(t, x)$ can be expressed in terms of what we call generalized maximum packings for $\tau^{(i)}$. We say that $\sigma \in S_{2 n}$ is a generalized maximum packing for $\tau^{(i)}$ if we can break $\sigma$ into consecutive blocks $\sigma=B_{1} \ldots B_{k}$ such that

1. for all $1 \leqslant j \leqslant k, B_{j}$ is either an increasing sequence of length 2 or $\operatorname{red}\left(B_{j}\right)$ is a maximum packing for $\tau^{(i)}$ of length $2 s$ for some $s \geqslant 2$ and

2. for all $1 \leqslant j \leqslant k-1$, the last element of $B_{j}$ is less than the first element of $B_{j+1}$.

Note that if $\sigma$ is a generalized maximum packing for $\tau^{(i)}$, there is only one possible block structure. That is, if $\sigma=\sigma_{1} \ldots \sigma_{2 n} \in S_{2 n}$ is a generalized maximum packing for $\tau^{(i)}$, our definitions force that $\sigma_{2 j-1}<\sigma_{2 j}$ for $i=1, \ldots, n$. Then it is easy to see that $\sigma_{2 j-1} \sigma_{2 j}$ and $\sigma_{2 j+1} \sigma_{2 j+2}$ are in the same block if and only if $\sigma_{2 j}>\sigma_{2 j+1}$.

If $\sigma$ is a generalized maximum packing for $\tau^{(i)}$ of length $2 n$ with block structure $B_{1} \ldots B_{k}$, then we define the weight $w\left(B_{j}\right)$ of block $B_{j}$ to be $(x-1)^{s}$ if $B_{j}$ has length $2 s+2$ where $s \geqslant 0$. Thus if $B_{i}$ is a block of length 2 , then $w\left(B_{i}\right)=1$. Then we define the weight $w(\sigma)$ of $\sigma$ to be $(-1)^{k-1} \prod_{j=1}^{k} w\left(B_{j}\right)$. For example,

$$
\sigma=123547698101112131415171618
$$

is a generalized maximum packing for $\tau^{(1)}=1324$ where $B_{1}=12, B_{2}=354769810$, $B_{3}=1112, B_{4}=1314$ and $B_{5}=15171618$. Thus $w\left(B_{1}\right)=w\left(B_{3}\right)=w\left(B_{4}\right)=1$, $w\left(B_{2}\right)=(x-1)^{3}$ and $w\left(B_{5}\right)=x-1$ so that $w(\sigma)=(-1)^{4}(x-1)^{4}=(x-1)^{4}$. Thus the weight of $\sigma$ is just $(-1)^{k-1}(x-1)^{\tau^{(i)}-\operatorname{mch}(\sigma)}$ where $k$ is the number of blocks of $\sigma$. We let $\mathcal{G M P}_{2 n, \tau^{(i)}}$ denote the set of $\sigma \in S_{2 n}$ which are generalized maximum packings for $\tau^{(i)}$ and we let

$$
G M P_{2 n, \tau^{(i)}}(x)=\sum_{\sigma \in \mathcal{G M P}} w(\sigma) .
$$

We say that $\sigma \in S_{2 n+1}$ is a generalized maximum packing for $\tau^{(i)}$ if we can break $\sigma$ into consecutive blocks $\sigma=B_{1} \ldots B_{k}$ such that

1. for all $1 \leqslant j<k, B_{j}$ is either an increasing sequence of length 2 or $\operatorname{red}\left(B_{j}\right)$ is a maximum packing of $\tau^{(i)}$ of length $2 s$ for some $s \geqslant 2$,

2. $B_{k}$ is a block of length 1 , and

3. for all $1 \leqslant j \leqslant k-1$, the last element of $B_{j}$ is less than the first element of $B_{j+1}$.

If $\sigma$ is a generalized maximum packing for $\tau^{(i)}$ of length $2 n+1$ with block structure $B_{1} \ldots B_{k}$, then we let $w\left(B_{k}\right)=1$ and, for $j<k$, we let $w\left(B_{j}\right)=(x-1)^{s}$ if $B_{j}$ has length $2 s+2$. Then we let $w(\sigma)=(-1)^{k-1} \prod_{i=1}^{k} w\left(B_{i}\right)=(-1)^{k-1}(x-1)^{\tau^{(i)}-\mathrm{mch}(\sigma)}$. For example,

$$
\sigma=12354769810111213141517161819
$$


is a generalized maximum packing for $\tau^{(1)}=1324$ where $B_{1}=12, B_{2}=354769810$, $B_{3}=1112, B_{4}=1314$ and $B_{5}=15171618$, and $B_{6}=19$. Thus $w\left(B_{1}\right)=w\left(B_{3}\right)=$ $w\left(B_{4}\right)=w\left(B_{6}\right)=1, w\left(B_{2}\right)=(x-1)^{3}$ and $w\left(B_{5}\right)=x-1$ so that $w(\sigma)=(-1)^{5}(x-1)^{4}=$ $-(x-1)^{4}$. We let $\mathcal{G M P}_{2 n+1, \tau^{(i)}}$ denote the set of $\sigma \in S_{2 n+1}$ which are generalized maximum packings for $\tau^{(i)}$. We then let

$$
\operatorname{GMP}_{2 n+1, \tau^{(i)}}(x)=\sum_{\sigma \in \mathcal{G} \mathcal{M P}} w(\sigma) .
$$

We shall call $G M P_{n, \tau^{(i)}}(x)$ the generalized maximum packing polynomial for $\tau^{(i)}$ of length $n$.

In general, it is much more difficult to compute $G M P_{2 n, \tau^{(i)}}(x)$ and $G M P_{2 n+1, \tau^{(i)}}(x)$ than to compute $\mathrm{mp}_{2 n, \tau^{(i)}}$ and $\mathrm{mp}_{2 n+1, \tau^{(i)}}$. Indeed, we do not have a closed expression for either $G M P_{2 n, \tau^{(i)}}(x)$ or $G M P_{2 n+1, \tau^{(i)}}(x)$ as a function of $n$ for any $i$. However, we will show that for $i \in\{1,2,4\}, G M P_{n, \tau^{(i)}}(x)$ can be computed via simple recursions. For example, we shall show that $G M P_{2, \tau^{(1)}}(x)=1, G M P_{4, \tau^{(1)}}(x)=x-2$, and, for $2 n>4$,

$$
G M P_{2 n, \tau^{(1)}}(x)=C_{n-1}(x-1)^{n-1}-\sum_{k=1}^{n-1} C_{k-1}(x-1)^{k-1} G M P_{2 n-2 k, \tau^{(1)}}(x) .
$$

Moreover, we shall show that $G M P_{1, \tau^{(1)}}(x)=1$ and $G M P_{2 n+1, \tau^{(1)}}(x)=-G M P_{2 n, \tau^{(1)}}(x)$ for $n \geqslant 1$.

Our main theorem is the following.

Theorem 1. For $\tau \in \mathcal{A}_{4}$,

$$
\begin{gathered}
A_{\tau}(t, x)=\frac{1}{1-\sum_{n \geqslant 1} G M P_{2 n, \tau}(x) \frac{t^{2 n}}{(2 n) !}} \text { and } \\
B_{\tau}(t, x)=\frac{\sum_{n \geqslant 1} G M P_{2 n-1, \tau}(x) \frac{t^{2 n-1}}{(2 n-1) !}}{1-\sum_{n \geqslant 1} G M P_{2 n, \tau}(x) \frac{t^{2 n}}{(2 n) !}}
\end{gathered}
$$

We shall prove Theorem 1 by applying the so-called homomorphism method which has been developed in a series of papers $[1,3,4,13,15,21,22,24,26,27]$. In particular, we shall show that the generating functions in Theorem 1 arise by applying certain ring homomorphisms defined on the ring of symmetric functions $\Lambda$ in infinitely many variables to simple symmetric function identities. For example, let $h_{n}$ denote the $n$th homogeneous symmetric function in $\Lambda$ and $e_{n}$ denote the $n$th elementary symmetric function in $\Lambda$. That is, $h_{n}$ and $e_{n}$ are defined by the generating functions

$$
H(t):=\sum_{n \geqslant 0} h_{n} t^{n}=\prod_{i} \frac{1}{1-x_{i} t} \text { and } E(t):=\sum_{n \geqslant 0} e_{n} t^{n}=\prod_{i}\left(1+x_{i} t\right) .
$$

Then we shall show that (6) arises by applying a ring homomorphism $\theta$ to the simple symmetric function identity

$$
H(t)=\frac{1}{E(-t)}
$$


For example, we shall show that

$$
(2 n) ! \theta\left(h_{2 n}\right)=A_{2 n, \tau^{(i)}}(x)=\sum_{\sigma \in \mathcal{A}_{2 n}} x^{\tau^{(i)}-\operatorname{mch}(\sigma)}
$$

for an appropriately chosen ring homomorphism $\theta$. Typically, one proves equations like (9) by interpreting the left-hand side of (9) in terms of a signed weighted sum of filled brick tabloids and then applying an appropriate sign-reversing weight-preserving involution to show that the combinatorial interpretation of $(2 n) ! \theta\left(h_{2 n}\right)$ reduces to the desired polynomial. The situation in this paper is a bit different from previous examples of the homomorphism method in that it requires two involutions to show that our combinatorial interpretation of $(2 n) ! \theta\left(h_{2 n}\right)$ reduces to the right-hand of (9). Equation (7) is proved in a similar manner except that we apply $\theta$ to a more complicated symmetric function identity.

The outline of the paper is as follows. In Section 2, we shall provide the necessary background on symmetric functions that is required for our proofs. In Section 3, we shall prove Theorem 1. In Section 4, we shall show how to compute $\operatorname{mp}_{n, \tau^{(i)}}$ for $i=1,2,3,4,5$. In Section 5, we shall develop recursions for $G M P_{n, \tau^{(i)}}(x)$ for $i=1,2,4$. The simplest case is the set of the recursions for $G M P_{n, \tau^{(1)}}(x)$ described above. In that case, we shall show that $G M P_{2 n, \tau^{(1)}}(0)=(-1)^{n-1} C_{n}$ for $n \geqslant 1$ and $\left.G M P_{2 n, \tau^{(1)}}(x)\right|_{x}=(-1)^{n}\left(\begin{array}{c}2 n \\ n-2\end{array}\right)$ where for any formal power series $f(x)=\sum_{n \geqslant 0} f_{n} x^{n}$, we write $\left.f(x)\right|_{x^{n}}$ the coefficient of $x^{n}$ in $f$. Using these facts, we can compute the generating functions for the number of up-down permutations with no $\tau^{(1)}$-matches or with exactly one $\tau^{(1)}$-match. For example, we shall show that

$$
1+\sum_{n \geqslant 1} \frac{t^{2 n}}{(2 n) !} N_{2 n, \tau^{(1)}}=\frac{1}{1+\sum_{n \geqslant 1}(-1)^{n} C_{n} \frac{t^{2 n}}{(2 n) !}}
$$

where $N_{2 n, \tau^{(1)}}$ is the number of $\sigma \in \mathcal{A}_{2 n}$ with no $\tau^{(1)}$-matches. Finally, in Section 6, we shall study the distribution of double rise pairs and double descent pairs in up-down permutations. That is, if $\sigma=\sigma_{1} \ldots \sigma_{n} \in \mathcal{A}_{n}$ is an up-down permutation, then we say that a pair $(2 i-1)(2 i)$ is a double rise pair if both $\sigma_{2 i-1}<\sigma_{2 i+1}$ and $\sigma_{2 i}<\sigma_{2 i+2}$. Thus $(2 i-1)(2 i)$ is a double rise pair in $\sigma$ if and only if there is a 1324 match starting at position $2 i-1$ in $\sigma$. We say that a pair $(2 i-1)(2 i)$ is a double descent pair if both $\sigma_{2 i-1}>\sigma_{2 i+1}$ and $\sigma_{2 i}>\sigma_{2 i+2}$. Thus $(2 i-1)(2 i)$ is a double descent pair in $\sigma$ if and only if there is a $D$-match starting at position $2 i-1$ in $\sigma$ where $D=\{2413,3412\}$.

\section{Symmetric Functions}

In this section we give the necessary background on symmetric functions needed for our proofs of the generating functions (6) and (7).

Let $\Lambda$ denote the ring of symmetric functions over infinitely many variables $x_{1}, x_{2}, \ldots$ with coefficients in some field $F$. We let $\Lambda_{n}$ denote the space of homogeneous symmetric functions of degree $n$ so that $\Lambda=\oplus_{n \geqslant 0} \Lambda_{n}$.

Let $\lambda=\left(\lambda_{1}, \ldots, \lambda_{\ell}\right)$ be an integer partition, that is, $\lambda$ is a finite sequence of weakly increasing non-negative integers. Let $\ell(\lambda)$ denote the number of nonzero integers in $\lambda$. 
If the sum of these integers is $n$, we say that $\lambda$ is a partition of $n$ and write $\lambda \vdash n$. For any partition $\lambda=\left(\lambda_{1}, \ldots, \lambda_{\ell}\right)$, let $e_{\lambda}=e_{\lambda_{1}} \cdots e_{\lambda_{\ell}}$ and $h_{\lambda}=h_{\lambda_{1}} \cdots h_{\lambda_{\ell}}$. The well-known fundamental theorem of symmetric functions says that $\left\{e_{\lambda}: \lambda\right.$ is a partition $\}$ is a basis for $\Lambda$ or, equivalently, that $\left\{e_{0}, e_{1}, \ldots\right\}$ is an algebraically independent set of generators for $\Lambda$. Since $\left\{e_{0}, e_{1}, \ldots\right\}$ is an algebraically independent set of generators for $\Lambda$, we can specify a ring homomorphism $\theta$ on $\Lambda$ by simply defining $\theta\left(e_{n}\right)$ for all $n \geqslant 0$.

A brick tabloid of shape $(n)$ and type $\lambda=\left(\lambda_{1}, \ldots, \lambda_{k}\right)$ where $\lambda \vdash n$ is a filling of a row of $n$ squares of cells with bricks of lengths $\lambda_{1}, \ldots, \lambda_{k}$ such that bricks do not overlap. For example, if $\lambda=\left(1^{2}, 2^{2}\right)$, the six $\lambda$-brick tabloids of shape (6) are pictured in Figure 1.

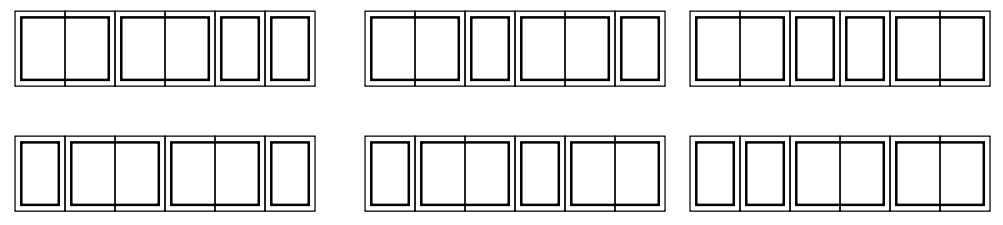

Figure 1: The six brick tabloids of type $\left(1^{2}, 2^{2}\right)$ and shape (6).

Let $\mathcal{B}_{\lambda, n}$ denote the set of all $\lambda$-brick tabloids of shape $(n)$ and let $B_{\lambda, n}=\left|\mathcal{B}_{\lambda, n}\right|$. We shall write $B=\left(b_{1}, \ldots, b_{k}\right)$ if $B$ is a brick tabloid of shape $n$ such that the lengths of the bricks in $B$ are $b_{1}, \ldots, b_{k}$ as we read from left to right. Eğecioğlu and Remmel proved in [9] that

$$
h_{n}=\sum_{\lambda \vdash n}(-1)^{n-\ell(\lambda)} B_{\lambda, n} e_{\lambda}=\sum_{\lambda \vdash n}(-1)^{n-\ell(\lambda)} \sum_{\left(b_{1}, \ldots, b_{\ell(\mu)}\right) \in \mathcal{B}_{\lambda, n}} \prod_{i=1}^{\ell(\mu)} e_{b_{i}} .
$$

Next we define a class of symmetric functions $p_{n, \nu}$ which have a relationship with $e_{\lambda}$ that is analogous to the relationship between $h_{n}$ and $e_{\lambda}$. These functions were first introduced in [17] and [21]. Let $\nu$ be a function which maps the set of non-negative integers into the field $F$. Recursively define $p_{n, \nu} \in \Lambda_{n}$ by setting $p_{0, \nu}=1$ and

$$
p_{n, \nu}=(-1)^{n-1} \nu(n) e_{n}+\sum_{k=1}^{n-1}(-1)^{k-1} e_{k} p_{n-k, \nu} \text { for all } n \geqslant 1 .
$$

By multiplying series, this means that

$$
\left(\sum_{n \geqslant 0}(-1)^{n} e_{n} t^{n}\right)\left(\sum_{n \geqslant 1} p_{n, \nu} t^{n}\right)=\sum_{n \geqslant 1}\left(\sum_{k=0}^{n-1} p_{n-k, \nu}(-1)^{k} e_{k}\right) t^{n}=\sum_{n \geqslant 1}(-1)^{n-1} \nu(n) e_{n} t^{n},
$$

where the last equality follows from the definition of $p_{n, \nu}$. Therefore,

$$
\sum_{n \geqslant 1} p_{n, \nu} t^{n}=\frac{\sum_{n \geqslant 1}(-1)^{n-1} \nu(n) e_{n} t^{n}}{\sum_{n \geqslant 0}(-1)^{n} e_{n} t^{n}}
$$


or, equivalently,

$$
1+\sum_{n \geqslant 1} p_{n, \nu} t^{n}=\frac{1+\sum_{n \geqslant 1}(-1)^{n}\left(e_{n}-\nu(n) e_{n}\right) t^{n}}{\sum_{n \geqslant 0}(-1)^{n} e_{n} t^{n}}
$$

When taking $\nu(n)=1$ for all $n \geqslant 1$, (13) becomes

$$
1+\sum_{n \geqslant 1} p_{n, 1} t^{n}=1+\frac{\sum_{n \geqslant 1}(-1)^{n-1} e_{n} t^{n}}{\sum_{n \geqslant 0}(-1)^{n} e_{n} t^{n}}=\frac{1}{\sum_{n \geqslant 0}(-1)^{n} e_{n} t^{n}}=1+\sum_{n \geqslant 1} h_{n} t^{n}
$$

which implies that $p_{n, 1}=h_{n}$ for all $n$. Other special cases for $\nu$ give well-known generating functions. For example, if $\nu(n)=n$ for $n \geqslant 1$, then $p_{n, \nu}$ is the power symmetric function $p_{n}=\sum_{i} x_{i}^{n}$. For any statement $A$, we let $\chi(A)=1$ if $A$ is true and $\chi(A)=0$ if $A$ is false. If $\nu(n)=(-1)^{k} \chi(n \geqslant k+1)$ for some $k \geqslant 1$, then $p_{n, \nu}$ is the Schur function $s_{\left(1^{k}, n-k\right)}$ corresponding to the partition $\left(1^{k}, n\right)$.

The coefficient of $e_{\lambda}$ in $p_{n, \nu}$ has a nice combinatorial interpretation similar to that of $h_{n}$. Suppose $T$ is a brick tabloid of shape $(n)$ and type $\lambda$ and that the final brick in $T$ has length $\ell$. Define the weight of a brick tabloid $w_{\nu}(T)$ to be $\nu(\ell)$ and let

$$
w_{\nu}\left(B_{\lambda, n}\right)=\sum_{T \in \mathcal{B}_{\lambda, n}} w_{\nu}(T) .
$$

It was proved in [17] and [21] that

$$
p_{n, \nu}=\sum_{\lambda \vdash n}(-1)^{n-\ell(\lambda)} w_{\nu}\left(B_{\lambda, n}\right) e_{\lambda}=\sum_{\lambda \vdash n}(-1)^{n-\ell(\lambda)} \sum_{\left(b_{1}, \ldots, b_{\ell(\mu)}\right) \in \mathcal{B}_{\lambda, n}} \nu\left(b_{\ell(\mu)}\right) \prod_{i=1}^{\ell(\mu)} e_{b_{i}} .
$$

\section{The proof of Theorem 1}

In this section, we shall prove Theorem 1. Fix $\tau \in \mathcal{A}_{4}$.

We start out by proving (6). Define a ring homomorphism $\theta$ from $\Lambda$ into $\mathbb{Q}(x)$ by setting $\theta\left(e_{0}\right)=1, \theta\left(e_{2 n+1}\right)=0$ for all $n \geqslant 0$, and

$$
\theta\left(e_{2 n}\right)=\frac{(-1)^{2 n-1}}{(2 n) !} G M P_{2 n, \tau}(x) \text { for all } n \geqslant 1 .
$$

Then we claim that $\theta\left(h_{2 n-1}\right)=0$ and

$$
(2 n) ! \theta\left(h_{2 n}\right)=\sum_{\sigma \in \mathcal{A}_{2 n}} x^{\tau-\operatorname{mch}(\sigma)}
$$

for all $n \geqslant 1$. Note that by (10),

$$
\theta\left(h_{2 n-1}\right)=\sum_{\mu \vdash 2 n-1}(-1)^{2 n-1-\ell(\mu)} B_{\mu, 2 n-1} \theta\left(e_{\mu}\right) .
$$


Clearly if $\mu$ is a partition of $2 n-1$, then $\mu$ must have an odd part so that $\theta\left(e_{\mu}\right)=0$. Thus $\theta\left(h_{2 n-1}\right)=0$ for all $n \geqslant 1$. Note also that

$$
\theta\left(h_{2 n}\right)=\sum_{\mu \vdash 2 n}(-1)^{2 n-\ell(\mu)} \sum_{\left(b_{1}, \ldots, b_{\ell(\mu)}\right) \in \mathcal{B}_{\mu, 2 n}} \prod_{i=1}^{\ell(\mu)} \theta\left(e_{b_{i}}\right)
$$

so that there is no loss if we restrict the sum on the right-hand side of (17) to partitions $\mu$ where every part of $\mu$ is even, i.e., to partitions of the form $2 \lambda$ where $\lambda$ is a partition of $n$ and $2 \lambda=\left(2 \lambda_{1}, \ldots, 2 \lambda_{\ell(\lambda)}\right)$ if $\lambda=\left(\lambda_{1}, \ldots, \lambda_{\ell(\lambda)}\right)$. Thus

$$
\begin{aligned}
(2 n) ! \theta\left(h_{2 n}\right) & =(2 n) ! \sum_{\lambda \vdash n}(-1)^{2 n-\ell(\lambda)} \sum_{\left(2 b_{1}, \ldots, 2 b_{\ell(\lambda)}\right) \in \mathcal{B}_{2 \lambda, n}} \prod_{j=1}^{\ell(\mu)} \theta\left(e_{2 b_{j}}\right) \\
& =(2 n) ! \sum_{\lambda \vdash n}(-1)^{2 n-\ell(\lambda)} \sum_{\left(2 b_{1}, \ldots, 2 b_{\ell(\lambda)}\right) \in \mathcal{B}_{2 \lambda, n}} \prod_{j=1}^{\ell(\mu)} \frac{(-1)^{2 b_{j}-1}}{\left(2 b_{j}\right) !} G M P_{2 b_{j}, \tau}(x) \\
& =\sum_{\lambda \vdash n} \sum_{T=\left(2 b_{1}, \ldots, 2 b_{\ell(\lambda)}\right) \in \mathcal{B}_{2 \lambda, 2 n}}\left(\begin{array}{c}
2 n \\
2 b_{1}, \ldots, 2 b_{\ell(\lambda)}
\end{array}\right) \prod_{j=1}^{\ell(\lambda)} G M P_{2 b_{j}, \tau}(x) .
\end{aligned}
$$

Next we want to give a combinatorial interpretation to the right-hand side of (18). We start with a brick tabloid $T=\left(2 b_{1}, \ldots, 2 b_{\ell(\lambda)}\right)$ of type $2 \lambda$. Then the binomial coefficient $\left(\begin{array}{c}2 n \\ 2 b_{1}, \ldots, 2 b_{\ell(\lambda)}\end{array}\right)$ allows us to pick a set partition $\vec{U}=\left(U_{1}, \ldots, U_{\ell(\lambda)}\right)$ of $\{1, \ldots, 2 n\}$ where $\left|U_{i}\right|=2 b_{i}$ for $i=1, \ldots, \ell(\lambda)$. Next we use the factor $\prod_{j=1}^{\ell(\lambda)} G M P_{2 b_{j}, \tau}(x)$ to choose a sequence of permutations $\vec{\sigma}=\left(\sigma^{(1)}, \ldots, \sigma^{(\ell(\lambda))}\right)$ such that $\sigma^{(j)} \in S_{2 \lambda_{j}}$ is a generalized maximum packing for $\tau$ for $j=1, \ldots, \ell(\lambda)$. Then for each $j$, we let $\alpha^{(j)}$ be the sequence that arises by replacing the $r$ th largest element of $\sigma^{(j)}$ by the $r$ th largest element of $U_{j}$ and then we place the elements of $\alpha^{(j)}$ in the cells of brick $2 b_{j}$ from left to right. For example, we have illustrated this process in Figure 2 for $\tau=\tau^{(1)}=1324$ where the brick tabloid is $T=(2,8,6)$. We have also indicated the block structure in each brick by underlining those elements in a common block. The weight $w(T, \vec{U}, \vec{\sigma})$ of such a triple $(T, \vec{U}, \vec{\sigma})$ is $\prod_{j=1}^{\ell(\lambda)} w\left(\sigma^{(j)}\right)$. We can interpret $w(T, \vec{U}, \vec{\sigma})$ as $\prod_{j=1}^{2 n} L(j)$ where $L:\{1, \ldots, 2 n\} \rightarrow \mathbb{Q}[x]$ is a labeling of the cells of $T$ which is defined as follows. First we define a labeling $\bar{L}:\{1, \ldots, 2 n\} \rightarrow \mathbb{Q}[x]$ where $\bar{L}(j)=1$ if cell $j$ does not start a $\tau$-match that is contained in its brick and $\bar{L}(j)=x-1$ if cell $j$ starts a $\tau$-match that is contained in its brick. Then we define $L(j)=-\bar{L}(j)$ if $j$ is the first cell of its block and that block is not the last block in its brick and $L(j)=\bar{L}(j)$ otherwise. Thus the RHS of (18) can be interpreted as the sum of the weights of all triples $(T, \alpha, L)$ such that

1. $T=\left(d_{1}, \ldots, d_{k}\right)$ is a brick tabloid of shape $(2 n)$ where each brick $d_{j}$ has even length,

2. $\alpha$ is a permutation of $S_{2 n}$ such that in each brick $d_{j}$, the sequence of elements in brick $d_{j}$ reduces to a permutation in $\mathcal{G} \mathcal{M P}_{d_{j}, \tau}$, and 
3. $L:\{1, \ldots, 2 n\} \rightarrow \mathbb{Q}[x]$ is the labeling of the cells of $T$ described above.

For example, in Figure 2, $T=(2,8,6), \alpha=13457109121113286141516$, and $L$ is the labeling where all the cells which do not have an explicit label in them are assumed to have label 1 .

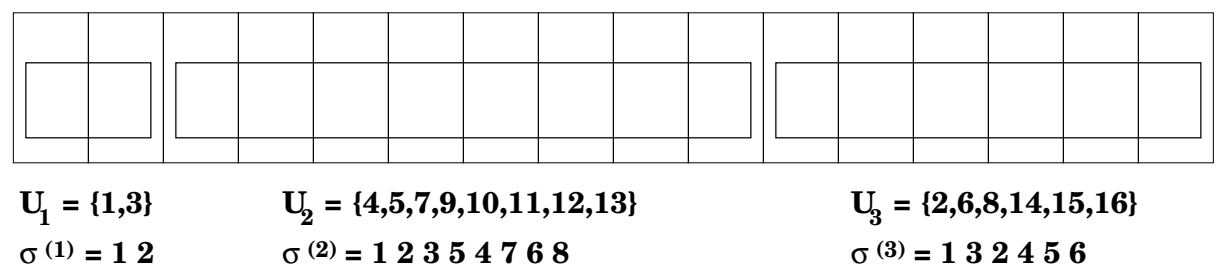

\begin{tabular}{|l|l|l|l|l|l|l|l|l|l|l|l|l|l|l|l|}
\hline 1 & & -1 & & $(x-1)$ & & $(x-1)$ & & & & $\mid-(x-1)$ & & & & 1 & \\
\hline 1 & $\mathbf{3}$ \\
\hline
\end{tabular} \mid \begin{tabular}{|l|c|c|c|c|c|c|c|c|}
\hline $\mathbf{4}$ & $\mathbf{5}$ & $\mathbf{7}$ & $\mathbf{1 0}$ & $\mathbf{9}$ & $\mathbf{1 2}$ & $\mathbf{1 1}$ & $\mathbf{1 3}$ \\
\hline
\end{tabular}

Figure 2: An element of $\mathcal{T}_{16, \tau^{(1)}}$

We let $\mathcal{T}_{2 n, \tau}$ denote the set of all such triples constructed in this way. It then follows that

$$
(2 n) ! \theta\left(h_{2 n}\right)=\sum_{(T, \alpha, L) \in \mathcal{T}_{2 n, \tau}} w(T, \alpha, L) .
$$

Next we will define two involutions $I$ and $J$ which will show that right-hand side of (19) is equal to the right-hand side of (16). We define $I: \mathcal{T}_{2 n, \tau} \rightarrow \mathcal{T}_{2 n, \tau}$ as follows. Suppose that we are given a triple $(T, \alpha, L)$ where $T=\left(d_{1}, \ldots, d_{k}\right)$. Then read the bricks from left to right until you find the first brick $d_{j}$ such that either (i) the generalized maximum packing corresponding to the elements in $d_{j}$ consists of more than one block or (ii) the generalized maximum packing corresponding to the elements in $d_{j}$ consists of a single block and the last element of $d_{j}$ is less than the first element of the following brick $d_{j+1}$. In case (i), split $d_{j}$ into two bricks $d^{*}$ and $d^{* *}$ where $d^{*}$ contains the cells of the first block in the generalized maximum packing corresponding to the elements in $d_{j}$ and $d^{* *}$ contains the remaining cells of $d_{j}$. We keep all the labels the same except that we change the label on the first cell of $d^{*}$ from -1 to 1 if the first block of $d_{j}$ is of length 2 and from $-(x-1)$ to $(x-1)$ if the first block of $d_{j}$ has length $\geqslant 4$. In case (ii), we combine bricks $d_{j}$ and $d_{j+1}$ into a single brick $d$. Note that since the last element of $d_{j}$ is less than the first element of $d_{j+1}$, the elements in the new brick $d$ will still reduce to a generalized maximum packing for $\tau$. We keep all the labels the same except that we change the label on the first cell of $d_{j}$ from 1 to -1 if $d_{j}$ is of length 2 and from $(x-1)$ to $-(x-1)$ if $d_{j}$ has length $\geqslant 4$. In both cases, we do not change the underlying permutation $\alpha$. If neither case (i) nor case (ii) applies, then $I(T, \alpha, L)=(T, \alpha, L)$. For example, if $(T, \alpha, L)$ is the element of $\mathcal{T}_{16, \tau^{(1)}}$ pictured in Figure 2, then we are in case (ii) since we can combine the first and second bricks so that $I(T, \alpha, L)$ is pictured in Figure 3. 


\begin{tabular}{|c|c|c|c|c|c|c|c|c|c|c|c|c|c|c|c|}
\hline-1 & & -1 & & $(x-1)$ & & $(x-1)$ & & & & $-(x-1)$ & & & & 1 & \\
\hline $\mathbf{1}$ & $\mathbf{3}$ & $\mathbf{4}$ & $\mathbf{5}$ & $\mathbf{7}$ & $\mathbf{1 0}$ & $\mathbf{9}$ & $\mathbf{1 2}$ & $\mathbf{1 1}$ & $\mathbf{1 3}$ \\
\hline
\end{tabular}

Figure 3: The image of $(T, \alpha, L)$ in Figure 2 under $I$.

It is easy to see that if $I(T, \alpha, L)=\left(T^{\prime}, \alpha, L^{\prime}\right) \neq(T, \alpha, L)$, then $I\left(T^{\prime}, \alpha, L^{\prime}\right)=(T, \alpha, L)$ and $w(T, \alpha, L)=-w\left(T^{\prime}, \alpha, L^{\prime}\right)$. Hence $I$ shows that

$$
\begin{aligned}
(2 n) ! \theta\left(h_{2 n}\right) & =\sum_{\substack{(T, \alpha, L) \in \mathcal{T}_{2 n, \tau}\\
}} w(T, \alpha, L) \\
& \sum_{\substack{(T, \alpha, L) \in \mathcal{T}_{2 n, \tau} \\
I(T, \alpha, L)=(T, \alpha, L)}} w(T, \alpha, L) .
\end{aligned}
$$

Thus we must examine the fixed points of $I$. Clearly, if $(T, \alpha, L)$ is a fixed point of $I$, then the elements of each brick $d$ in $T$ must reduce to a generalized maximum packing of $\tau$ which consists of a single block. Second, we must not be able to combine any two bricks so that if $T=\left(d_{1}, \ldots, d_{k}\right)$, then the last element of $d_{j}$ is greater than the first element of $d_{j+1}$ for $j=1, \ldots, k-1$. But this means that the underlying permutation $\alpha$ is an up-down permutation. It follows that the fixed points of $I$ consists of triples $(T, \alpha, L)$ such that

(I) $\alpha$ is an up-down permutation of length $2 n$,

(II) $T=\left(d_{1}, \ldots, d_{k}\right)$ where each $d_{j}$ has even length and the elements of $d_{j}$ reduce to a generalized maximum packing of $\tau$ which consists of a single block, and

(III) the label of $L(j)$ of the $j$ th cell of $T$ is $(x-1)$ if $j$ is the start of $\tau$-match in $\alpha$ that lies in its brick and is equal to 1 otherwise.

Next we want to modify our interpretation of the right-hand side of (20) to consist of all triples $\left(T^{\prime}, \alpha, L^{\prime}\right)$ such that

$\left(I^{\prime}\right) \alpha$ is an up-down permutation of length $2 n$,

$\left(I I^{\prime}\right) T=\left(d_{1}, \ldots, d_{k}\right)$ where each $d_{j}$ has even length and the elements of $d_{j}$ reduce to a generalized maximum packing of $\tau$ which consists of a single block, and

$\left(I I I^{\prime}\right)$ the label of $L(j)$ of the $j$ th cell of $T$ is either $x$ or -1 if $j$ is the start of $\tau$-match in $\alpha$ that lies in its brick and is equal to 1 otherwise.

We let $\mathcal{F} \mathcal{I}_{2 n, \tau}$ denote the set of triples $\left(T^{\prime}, \alpha, L^{\prime}\right)$ satisfying $\left(I^{\prime}\right)-\left(I I I^{\prime}\right)$. Then for any $\left(T^{\prime}, \alpha, L^{\prime}\right) \in \mathcal{F} \mathcal{I}_{2 n, \tau}$, we define the weight $w\left(T^{\prime}, \alpha, L^{\prime}\right)$ of $\left(T^{\prime}, \alpha, L^{\prime}\right)$ to be $\prod_{j=1}^{2 n} L^{\prime}(j)$. For example, Figure 4 pictures an element of $\mathcal{F I}_{16, \tau^{(1)}}$ whose weight is $x$, where again the cells which do not have labels are assumed to have label 1. 


\begin{tabular}{|l|l|l|l|l|l|l|l|l|l|l|l|l|l|l|l|}
\hline $\mathbf{1}$ & $\mathbf{7}$ \\
\hline
\end{tabular}

Figure 4: An element of $\mathcal{F} \mathcal{I}_{16, \tau^{(1)}}$.

It then follows that

$$
(2 n) ! \theta\left(h_{2 n}\right)=\sum_{\left(T^{\prime}, \alpha, L^{\prime}\right) \in \mathcal{F}_{2 n, \tau}} w\left(T^{\prime}, \alpha, L^{\prime}\right) .
$$

Next we define an involution $J: \mathcal{F I}_{2 n, \tau} \rightarrow \mathcal{F I}_{2 n, \tau}$. Given an element $(T, \alpha, L) \in$ $\mathcal{F} \mathcal{I}_{2 n, \tau}$, scan the cells of $T=\left(d_{1}, \ldots, d_{k}\right)$ from left to right looking for the first cell $c$ such that either (A) the label of $c$ is -1 or $(\mathrm{B}) c$ is the second to last element of a brick $d_{j}$ such that the elements of bricks $d_{j}$ and $d_{j+1}$ reduce to a generalized maximum packing of $\tau$ which consists of a single block. Note that in case (B), $c$ must have label 1 since it does not start match of $\tau$ in $\alpha$ that lies in its brick. In case $(\mathrm{A})$, if $c$ is in brick $d_{j}$, then break $d_{j}$ into two bricks $d^{*}$ and $d^{* *}$ where $d^{*}$ contain the cells of $d_{j}$ up to and including cell $c+1$ and $d^{* *}$ contains the rest of the cells of $d_{j}$. We then replace the -1 label on cell $c$ by 1 . In case (B), we replace the bricks $d_{j}$ and $d_{j+1}$ by a single brick $d$ and replace the label of 1 on $c$ by -1 . In either case, we do not change the underlying permutation $\alpha$. If neither case (A) nor case (B) applies, then we let $J(T, \alpha, L)=(T, \alpha, L)$. For example, if we consider the triple $(T, \alpha, L)$ pictured in Figure 4 , we cannot combine bricks $d_{1}$ and $d_{2}$ because $\alpha$ does not have a $\tau^{(1)}$-match starting cell 1 and we cannot combine bricks $d_{2}$ and $d_{3}$ because $\alpha$ does not have a $\tau^{(1)}$-match starting cell 3. Thus we are in case (B) where $d_{j}=d_{3}$ and $c=7$. Thus we split $d_{3}$ at cells 8 and 9 so that $J(T, \alpha, L)=\left(T^{\prime}, \alpha, L^{\prime}\right)$ is the filling pictured in Figure 5. Note that it will automatically be the case that the first action that we can take for $\left(T^{\prime}, \alpha, L^{\prime}\right)$ is to combine the two bricks that made up the $d_{3}$ in $(T, \alpha, L)$.

\begin{tabular}{|c|c|c|c|c|c|c|c|c|c|c|c|c|c|c|c|}
\hline & & & & $x$ & & & & & & -1 & & & & & \\
\hline 1 & 7 & 4 & 5 & $\underline{\mathbf{3}}$ & 10 & 9 & 12 & 11 & 13 & 2 & 8 & 6 & 14 & 15 & 16 \\
\hline
\end{tabular}

Figure 5: $J(T, \alpha, L)$ for $(T, \alpha, L)$ of Figure 4 .

It is easy to see that if $J(T, \alpha, L)=\left(T^{\prime}, \alpha, L^{\prime}\right) \neq(T, \alpha, L)$, then $w((T, \alpha, L)=$ $-w\left(T^{\prime}, \alpha, L^{\prime}\right)$ and $J\left(T^{\prime}, \alpha, L^{\prime}\right)=(T, \alpha, L)$. Thus it follows that

$$
(2 n) ! \theta\left(h_{2 n}\right)=\sum_{\substack{(T, \alpha, L) \in \mathcal{F} \mathcal{I}_{2 n, \tau} \\ J(T, \alpha, L)=(T, \alpha, L)}} w(T, \alpha, L) .
$$


Thus we must examine the fixed points of $J$. If $J(T, \alpha, L)=(T, \alpha, L)$, then clearly $(T, \alpha, L)$ can have no cells which have a -1 label. Thus in a brick $d$ of $T$ of length $\geqslant 4$, the start of every $\tau$-match contained in $d$ is labeled with an $x$. Moreover, we claim that there cannot be a $\tau$-match that involves cells in two different bricks $d_{j}$ and $d_{j+1}$. That is, the only way a $\tau$-match could span cells in both $d_{j}$ and $d_{j+1}$ is if that $\tau$ match started in cell $c$ which is the second to last cell of $d_{j}$. But this would imply that the elements of $d_{j}$ and $d_{j+1}$ would reduce to a generalized maximum packing for $\tau$ with a single block and, hence, case (B) of our involution would apply to $c$. Hence if $(T, \alpha, L)$ is a fixed point of $J$, then $w(T, \alpha, L)=x^{\tau-\operatorname{mch}(\alpha)}$.

For any $\alpha \in \mathcal{A}_{2 n}$, there is a unique fixed point $(T, \alpha, L)$ of $J$ whose underlying permutation is $\alpha$. That is, we must define the bricks $d_{1}, d_{2}, \ldots$ inductively as follows. We let $d_{1}$ be of length 2 if there is no $\tau$-match in $\alpha$ starting at 1 and $d_{1}$ be of length $2 s$ if there are $\tau$-matches starting at positions $1,3, \ldots, 2 s-3$ but not at $2 s-1$ in $\alpha$. Then having defined bricks $d_{1}, \ldots, d_{r}$ where $d_{r}$ ends at cell $c=2 k<2 n$, we let $d_{r+1}$ be of length 2 if there is no $\tau$-match in $\alpha$ starting at $2 k+1$ and $d_{r+1}$ be of length $2 s$ if there are $\tau$-matches starting at positions $2 k+1,2 k+3, \ldots, 2 k+2 s-3$ but not at $2 k+2 s-1$ in $\alpha$. Hence

$$
(2 n) ! \theta\left(h_{2 n}\right)=\sum_{\alpha \in \mathcal{A}_{2 n}} x^{\tau-\operatorname{mch}(\alpha)} .
$$

It then follows that

$$
\begin{aligned}
\theta\left(\sum_{n \geqslant 0} h_{n} t^{n}\right) & =1+\sum_{n \geqslant 1} \frac{t^{2 n}}{(2 n) !} \sum_{\alpha \in \mathcal{A}_{2 n}} x^{\tau-\operatorname{mch}(\alpha)} \\
& =\frac{1}{1+\sum_{n \geqslant 1}(-t)^{n} \theta\left(e_{n}\right)} \\
& =\frac{1}{1-\sum_{n \geqslant 1} \frac{t^{2 n}}{(2 n) !} G M P_{2 n, \tau}(x)}
\end{aligned}
$$

which is what we wanted to prove.

To prove (7), we will use the same ring homomorphism $\theta$ with weight function $\nu: \mathbb{P} \rightarrow$ $\mathbb{Q}(x)$ where

$$
\nu(2 n-1)=0 \text { and } \nu(2 n)=\frac{2 n G M P_{2 n-1, \tau}(x)}{G M P_{2 n, \tau}(x)} \text { for all } n \geqslant 1 .
$$

We have designed $\nu$ so that $\nu(2 n) \theta\left(e_{2 n}\right)=\frac{(-1)^{2 n-1}}{(2 n-1) !} G M P_{2 n-1, \tau}(x)$. Then we claim that for all $n \geqslant 0, \theta\left(p_{2 n+1, \nu}\right)=0$ and

$$
(2 n+1) ! \theta\left(p_{2 n+2, \nu}\right)=\sum_{\sigma \in \mathcal{A}_{2 n+1}} x^{\tau-\operatorname{mch}(\sigma)} .
$$

Note that by (14),

$$
\theta\left(p_{2 n+1, \nu}\right)=\sum_{\mu \vdash 2 n+1}(-1)^{2 n+1-\ell(\mu)} w_{\nu}\left(B_{\mu, 2 n+1}\right) \theta\left(e_{\mu}\right) .
$$


Clearly if $\mu$ is a partition of $2 n+1$, then $\mu$ must have an odd part so that $\theta\left(e_{\mu}\right)=0$. Thus $\theta\left(p_{2 n+1, \nu}\right)=0$ for all $n \geqslant 0$. It also follows that when we want to compute $\theta\left(p_{2 n+2, \nu}\right)$, we can restrict ourselves to considering partitions of the form $2 \lambda$ where $\lambda$ is a partition of $n+1$. Thus

$$
\begin{aligned}
& (2 n+1) ! \theta\left(p_{2 n+2, \nu}\right) \\
& =(2 n+1) ! \sum_{\lambda \vdash n+1}(-1)^{2 n+2-\ell(\lambda)} \sum_{\left(2 b_{1}, \ldots, 2 b_{\ell(\lambda)}\right) \in \mathcal{B}_{2 \lambda, 2 n+2}} \nu\left(2 b_{\ell(\lambda)}\right) \theta\left(e_{2 b_{\ell(\lambda)}}\right) \prod_{j=1}^{\ell(\lambda)-1} \theta\left(e_{2 b_{j}}\right) \\
& =(2 n+1) ! \sum_{\lambda \vdash n+1}(-1)^{2 n+2-\ell(\lambda)} \sum_{\left(2 b_{1}, \ldots, 2 b_{\ell(\lambda)}\right) \in \mathcal{B}_{2 \lambda, 2 n+2}} \frac{(-1)^{2 b_{\ell(\lambda)}-1}}{\left(2 b_{\ell(\lambda)}-1\right) !} G P M_{2 b_{\ell(\lambda)}-1, \tau}(x) \times \\
& =\prod_{\lambda \vdash n+1} \prod_{j=1}^{\ell(\lambda)-1} \frac{(-1)^{2 b_{j}-1}}{\left(2 b_{j}\right) !} G M P_{2 b_{j}, \tau}(x) \\
& \prod_{j=1}^{\ell(\lambda)-1} G M P_{2 b_{j}, \tau}(x) .
\end{aligned}
$$

As before, we want to give a combinatorial interpretation to the right-hand side of (24). We start with a brick tabloid $T=\left(2 b_{1}, \ldots, 2 b_{\ell(\lambda)}\right)$ of length $2 n+2$ and type

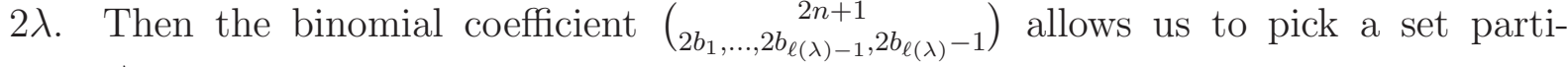
tion $\vec{U}=\left(U_{1}, \ldots, U_{\ell(\lambda)}\right)$ of $\{1, \ldots, 2 n+1\}$ where $\left|U_{i}\right|=2 b_{i}$ for $i=1, \ldots, \ell(\lambda)-1$ and $\left|U_{\ell(\lambda)}\right|=2 b_{\ell(\lambda)}-1$. Next we use the factor $G M P_{2 b_{\ell(\lambda)}-1, \tau}(x) \prod_{j=1}^{\ell(\lambda)-1} G M P_{2 b_{j}, \tau}(x)$ to choose a sequence of permutations $\vec{\sigma}=\left(\sigma^{(1)}, \ldots, \sigma^{(\ell(\lambda))}\right)$ such that $\sigma^{(j)} \in S_{2 b_{j}}$ is a generalized maximum packing for $\tau$ for $j=1, \ldots, \ell(\lambda)-1$ and $\sigma^{(\ell(\lambda))} \in S_{2 b_{\ell(\lambda)}-1}$ is a generalized maximum packing for $\tau$. Then for each $j$, we let $\alpha^{(j)}$ be the sequence that arises by replacing the $r$ th largest element of $\sigma^{(j)}$ by the $r$ th largest element of $U_{j}$ and then we place the elements of $\alpha^{(j)}$ in the cells of brick $2 b_{j}$ from left to right. This means that for the last brick $2 b_{\ell(\lambda)}$, we will fill in all but the last cell which we leave blank. For example, we have illustrated this process in Figure 6 for $\tau^{(1)}=1324$ where the underlying brick tableau $T=(2,8,6)$. We have also indicated the block structure in each brick by underlying those elements in a common block. The weight $w(T, \vec{U}, \vec{\sigma})$ of such a triple $(T, \vec{U}, \vec{\sigma})$ is $\prod_{j=1}^{\ell(\lambda)} w\left(\sigma^{(j)}\right)$. Again we can interpret $w(T, \vec{U}, \vec{\sigma})$ to be $\prod_{j=1}^{2 n+1} L(j)$ where $L:\{1, \ldots, 2 n\} \rightarrow \mathbb{Q}[x]$ is a labeling of the cells of $T$. To define $L$, we label the blank cell with 1 and then we label the remaining cells exactly as we did before. Thus the RHS of (24) can be interpreted as the sum of the weights of all triples $(T, \alpha, L)$ such that

1. $T=\left(d_{1}, \ldots, d_{k}\right)$ is a brick tabloid of shape $(2 n+2)$ where each brick $d_{j}$ has even length, 
2. $\alpha$ is a permutation of $S_{2 n+1}$ such that in each brick $d_{j}$ with $j<k$, the elements in brick $d_{j}$ reduce to a permutation in $\mathcal{G M} \mathcal{P}_{d_{j}, \tau}$ and the elements in brick $d_{k}$ fill the first $d_{k}-1$ cells of brick $d_{k}$ and reduce to a permutation in $\mathcal{G M P}_{d_{k}-1, \tau}$, and

3. $L:\{1, \ldots, 2 n\} \rightarrow \mathbb{Q}[x]$ is the labeling of the cells of $T$ described above.

For example, in Figure 6, $T=(2,8,6), \alpha=1437510912111328614$ 15, and $L$ is the labeling where all the cells which do not have an explicit label in them are assumed to have label 1.

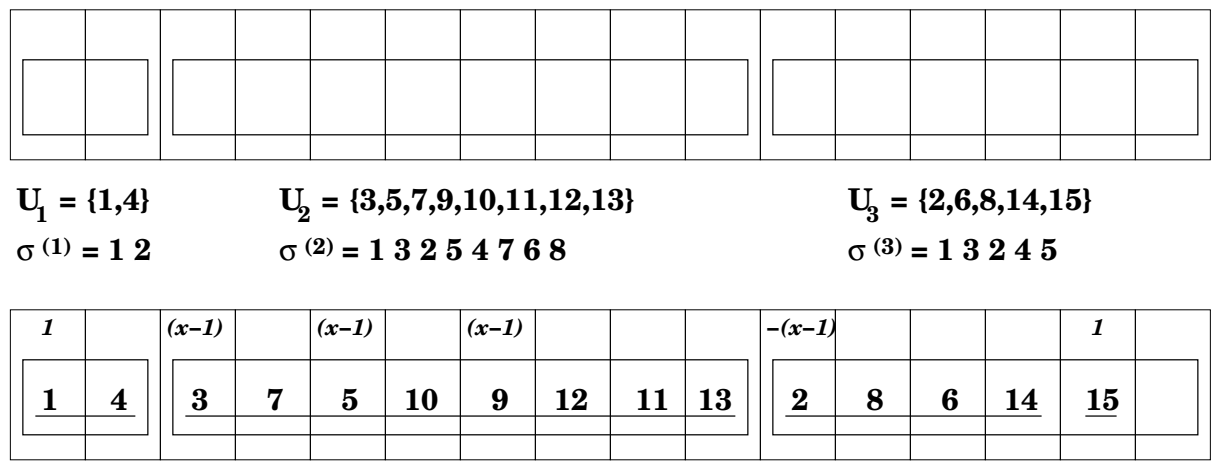

Figure 6: An element of $\mathcal{T}_{15, \tau^{(1)}}$.

We let $\mathcal{T}_{2 n+1, \tau}$ denote the set of all such triples constructed in this way. It then follows that

$$
(2 n+1) ! \theta\left(p_{2 n+2, \nu}\right)=\sum_{(T, \alpha, L) \in \mathcal{T}_{2 n+1, \tau}} w(T, \alpha, L) .
$$

Note that the only difference between the fillings of even length in the proof of (16) and our current fillings is that, in our current fillings, the last brick ends in a blank cell and the block structure of the reduction of the sequence of elements in the last brick must end in a block of size 1 . This means that we can define the two involutions $I$ and $J$ exactly as before since the fact that the last block of the final brick is length 1 does not change things. Using the same reasoning as in our proof of (16), it is easy to check that our involutions $I$ and $J$ show that

$$
(2 n+1) ! \theta\left(p_{2 n+2, \nu}\right)=\sum_{\alpha \in \mathcal{A}_{2 n+1}} x^{\tau-\operatorname{mch}(\alpha)} .
$$

It then follows that

$$
\begin{aligned}
\theta\left(\sum_{n \geqslant 1} p_{n, \nu} t^{n}\right) & =\sum_{n \geqslant 0} \frac{t^{2 n+2}}{(2 n+1) !} \sum_{\alpha \in \mathcal{A}_{2 n+1}} x^{\tau-\operatorname{mch}(\alpha)} \\
& =\frac{\sum_{n \geqslant 1}(-1)^{n-1} \nu(n) \theta\left(e_{n}\right) t^{n}}{\sum_{n \geqslant 0}(-1)^{n} \theta\left(e_{n}\right) t^{n}}
\end{aligned}
$$




$$
=\frac{\sum_{n \geqslant 1} \frac{t^{2 n}}{(2 n-1) !} G M P_{2 n-1, \tau}(x)}{1-\sum_{n \geqslant 1} \frac{t^{n}}{(2 n) !} G M P_{2 n, \tau}(x)} .
$$

Dividing the second and the last elements in the string of equalities in (27) by $t$ gives (7) which is what we wanted to prove.

\section{Computing $\mathbf{m p}_{n, \tau^{(i)}}$}

In this section, we shall consider the problem of computing $\mathrm{mp}_{\tau^{(i)}, n}$ since we will need such computations to compute $G M P_{\tau^{(i)}, n}$.

The problem of computing $\mathrm{mp}_{\tau^{(i)}, 2 n}$ has been studied by Harmse and Remmel [13] in a different context. Harmse and Remmel studied maximum packings in column strict arrays. That is, let $\mathcal{F}_{n, k}$ denote the set of all fillings of a $k \times n$ rectangular array with the integers $1, \ldots, k n$ such that the elements increase from bottom to top in each column. We let $(i, j)$ denote the cell in the $i$ th row from the bottom and the $j$ th column from the left of the $k \times n$ rectangle and we let $F(i, j)$ denote the element in cell $(i, j)$ of $F \in \mathcal{F}_{n, k}$.

If $F$ is any filling of a $k \times n$-rectangle with distinct positive integers such that elements in each column increase, reading from bottom to top, then we let $\operatorname{red}(F)$ denote the element of $\mathcal{F}_{n, k}$ which results from $F$ by replacing the $i$ th smallest element of $F$ by $i$. For example, Figure 7 demonstrates a filling, $F$, with its corresponding reduced filling, $\operatorname{red}(F)$.

$$
F=\begin{array}{|c|c|c|}
\hline 12 & 16 & 22 \\
\hline 8 & 15 & 17 \\
\hline 6 & 10 & 13 \\
\hline 1 & 7 & 5 \\
\hline
\end{array} \quad \operatorname{red}(F)=\begin{array}{|c|c|c|}
\hline 7 & 10 & 12 \\
\hline 5 & 9 & 11 \\
\hline 3 & 6 & 8 \\
\hline 1 & 4 & 2 \\
\hline
\end{array}
$$

Figure 7: An example of $F \in \mathcal{F}_{3,4}$ and $\operatorname{red}(F)$.

If $F \in \mathcal{F}_{n, k}$ and $1 \leqslant c_{1}<\cdots<c_{j} \leqslant n$, then we let $F\left[c_{1}, \ldots, c_{j}\right]$ be the filling of the $k \times j$ rectangle where the elements in column $a$ of $F\left[c_{1}, \ldots, c_{j}\right]$ equal the elements in column $c_{a}$ in $F$ for $a=1, \ldots, j$. If $P \in \mathcal{F}_{j, k}$ and $F \in \mathcal{F}_{n, k}$ where $j \leqslant n$, then we say there is a $P$-match in $F$ starting at position $i$ if $\operatorname{red}(F[i, i+1, \ldots, i+j-1])=P$. We let $P$-mch $(F)$ denote the number of $P$-matches in $F$. For example, if we consider the fillings $P \in \mathcal{F}_{3,3}$ and $F, G \in \mathcal{F}_{6,3}$ shown in Figure 8, then it is easy to see that there are no $P$-matches in $F$ and there are $2 P$-matches in $G$ starting at positions 1 and 2 so $P-\operatorname{mch}(F)=0$ and $P-\operatorname{mch}(G)=2$.

If $P \in \mathcal{F}_{2, k}$, then we define $\mathcal{M P}_{n}^{P}$ to be the set of $F \in \mathcal{F}_{n, k}$ with $P$-mch $(F)=n-1$, i.e. the set of $F \in \mathcal{F}_{n, k}$ with the property that there are $P$-matches in $F$ starting at positions $1,2, \ldots, n-1$. We let $\mathrm{mp}_{n}^{P}=\left|\mathcal{M P}_{n}^{P}\right|$ and, by convention, we define $\mathrm{mp}_{1}^{P}=1$.

Given an $F \in \mathcal{F}_{n, 2}$, we let $\sigma(F)$ be the permutation

$$
\sigma(F)=F(1,1) F(2,1) F(1,2) F(2,2) \ldots F(1, n) F(2, n) .
$$



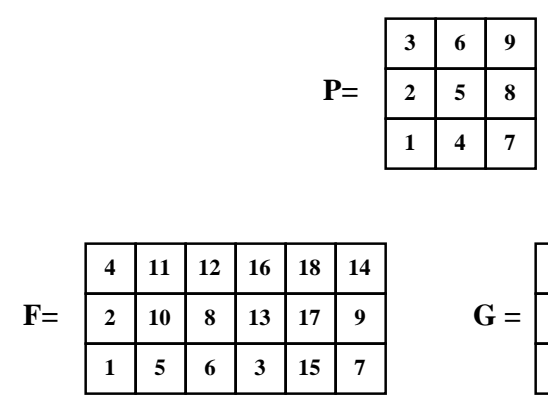

$\mathbf{G}=$\begin{tabular}{|c|c|c|c|c|c|}
\hline 4 & 7 & 11 & 16 & 18 & 14 \\
\hline 2 & 6 & 10 & 13 & 17 & 9 \\
\hline 1 & 5 & 8 & 12 & 15 & 3 \\
\hline
\end{tabular}

Figure 8: Computing the number of $P$-matches for elements in $\mathcal{F}_{6,3}$.

We then let $P^{(i)}$ denote the element of $\mathcal{F}_{2,2}$ such that $\sigma\left(P^{(i)}\right)=\tau^{(i)}$. For example, $P^{(1)}, \ldots, P^{(5)}$ are pictured in Figure 9 . It is then easy to see that for any maximum packing $F \in \mathcal{F}_{n, 2}$ of $P^{(i)}, \sigma(F)$ is an up-down permutation in $\mathcal{A}_{2 n}$ which is a maximum packing for $\tau^{(i)}$. Vice versa, if $\sigma=\sigma_{1} \ldots \sigma_{2 n}$ is a maximum packing of $\tau^{(i)}$, then the $2 \times n$ array $F_{\sigma}$ where $F_{\sigma}(1, i)=\sigma_{2 i-1}$ and $F_{\sigma}(2, i)=\sigma_{2 i}$ is a maximum packing for $P^{(i)}$. An example of this correspondence is pictured at the top of Figure 9 for $\tau^{(1)}=1324$ and $P^{(1)}=$\begin{tabular}{|l|l|}
\hline 3 & 4 \\
\hline 1 & 2 \\
\hline
\end{tabular}

$$
\begin{aligned}
& \begin{array}{|l|l|l|l|l|l|l|l|}
\hline 5 & 6 & 8 & 9 & 11 & 13 & 15 & 16 \\
\hline 1 & 2 & 3 & 4 & 7 & 10 & 12 & 14
\end{array} \longrightarrow 15263849711101312151416 \\
& \tau^{(1)}=1324 \longrightarrow \begin{array}{|l|l|}
\hline 3 & 4 \\
\hline 1 & 2 \\
\hline 1
\end{array}=P^{(1)} \tau^{(2)}=2314 \longrightarrow \begin{array}{|l|l|}
\hline 3 & 4 \\
\hline 2 & 1 \\
\hline
\end{array}=P^{(2)} \\
& \tau^{(3)}=2431 \longrightarrow \begin{array}{|l|l|}
\hline 4 & 3 \\
\hline 2 & 1 \\
\hline
\end{array}=P^{(3)} \tau^{(4)}=1432 \longrightarrow \begin{array}{|l|l|}
\hline 4 & 3 \\
\hline 1 & 2 \\
\hline
\end{array}=P^{(4)} \\
& \tau^{(5)}=3412 \longrightarrow \begin{array}{|l|l|}
\hline 4 & 2 \\
\hline 3 & 1 \\
\hline
\end{array}=P^{(5)}
\end{aligned}
$$

Figure 9: The correspondence between $\mathcal{M P}_{2 n, \tau^{(i)}}$ and $\mathcal{M P}_{n}^{P^{(i)}}$.

It follows that for $i=1, \ldots, 5, \operatorname{mp}_{2 n, \tau^{(i)}}=\operatorname{mp}_{n}^{P^{(i)}}$. Now Harmse and Remmel [13] proved that for $n \geqslant 2, \mathrm{mp}_{n}^{P^{(1)}}=\mathrm{mp}_{n}^{P^{(3)}}=C_{n-1}$ and $\mathrm{mp}_{n}^{P^{(2)}}=\mathrm{mp}_{n}^{P^{(4)}}=\mathrm{mp}_{n}^{P^{(5)}}=1$. Thus we obtain the following theorem.

Theorem 2. For all $n \geqslant 2, \operatorname{mp}_{2 n, \tau^{(1)}}=\operatorname{mp}_{2 n, \tau^{(3)}}=C_{n-1}$ and $\mathrm{mp}_{2 n, \tau^{(2)}}=\mathrm{mp}_{2 n, \tau^{(4)}}=\mathrm{mp}_{2 n, \tau^{(5)}}=1$. 
Our next goal is to prove the following.

Theorem 3. For all $n \geqslant 2, \mathrm{mp}_{2 n+1, \tau^{(1)}}=2 n C_{n-1}, \mathrm{mp}_{2 n+1, \tau^{(2)}}=2 n$, $\mathrm{mp}_{2 n+1, \tau^{(3)}}=C_{n-1}+C_{n}, \mathrm{mp}_{2 n+1, \tau^{(4)}}=n+1$ and $\mathrm{mp}_{2 n+1, \tau^{(5)}}=2$.

Proof. To compute $\mathrm{mp}_{2 n+1, \tau^{(i)}}$, we must exploit some of the techniques used by Harmse and Remmel [13] to compute $\operatorname{mp}_{n}^{P}$ for $P \in \mathcal{F}_{2, k}$. To help us visualize the order relationships within $P^{(i)}$, we form a directed graph $G_{P^{(i)}}$ on the cells of the $2 \times 2$ rectangle by drawing a directed edge from the position of the number $j$ to the position of the number $j+1$ in $P$ for $j=1,2,3$. For example, in Figure 10, the graph $G_{P^{(1)}}$ is pictured immediately to the right of $P^{(1)}$. Then $G_{P^{(i)}}$ determines the order relationships between all the cells in $P^{(i)}$ since $P^{(i)}(r, s)<P^{(i)}(u, v)$ if there is a directed path from cell $(r, s)$ to cell $(u, v)$ in $G_{P^{(i)}}$. Now suppose that $F \in \mathcal{M P}_{n}^{P^{(i)}}$ where $n \geqslant 3$. Because there is a $P^{(i)}$-match starting in column $j$ for each $1 \leqslant j<n$, we can superimpose $G_{P^{(i)}}$ on the cells in columns $j$ and $j+1$ to determine the order relations between the elements in those two columns. If we do this for every pair of columns, $j$ and $j+1$ for $j=1, \ldots, n-1$, we end up with a directed graph on the cells of the $2 \times n$ rectangle which we will call $G_{n, P^{(i)}}$. For example, in Figure $10, G_{6, P^{(1)}}$ is pictured in the second row. It is then easy to see that if $F \in \mathcal{M P}_{n}^{P^{(i)}}$ and there is a directed path from cell $(r, s)$ to cell $(u, v)$ in $G_{n, P^{(i)}}$, then it must be the case that $F(r, s)<F(u, v)$. Note that $G_{n, P^{(i)}}$ will always be a directed acyclic graph with no multiple edges.

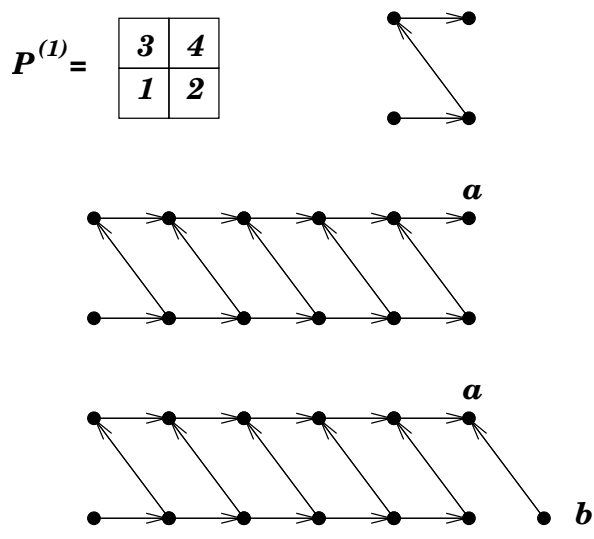

Figure 10: The graphs $G_{n, P^{(1)}}$ and $G_{n, P^{(1)}}^{+}$.

Harmse and Remmel [13] proved that the problem of computing $\mathrm{mp}_{n}^{P^{(i)}}$ for any $P^{(i)} \in$ $\mathcal{F}_{2,2}$ of shape $2^{2}$ can be reduced to finding the number of linear extensions of a certain poset associated with $P^{(i)}$. That is, the graph $G_{n, P^{(i)}}$ induces a poset $\mathcal{W}_{G_{n, P^{(i)}}}=(\{(i, j): 1 \leqslant i \leqslant$ $\left.2 \& 1 \leqslant j \leqslant n\},<_{W}\right)$ on the cells of the $2 \times n$ rectangle by defining $(i, j)<_{W}(s, t)$ if and only if there is a directed path from $(i, j)$ to $(s, t)$ in $G_{n, P^{(i)}}$. Harmse and Remmel proved that there is a 1:1 correspondence between the elements of $\mathcal{M P}_{n}$ and the linear extensions of $\mathcal{W}_{n, P^{(i)}}$. That is, if $F \in \mathcal{M P}_{n}^{P^{(i)}}$, then it is easy to see that $\left(a_{1}, b_{1}\right), \ldots,\left(a_{2 n}, b_{2 n}\right)$ where $F\left(a_{i}, b_{i}\right)=i$ is a linear extension of $\mathcal{W}_{G_{n, P}(i)}$. Vice versa, if $\left(a_{1}, b_{1}\right), \ldots,\left(a_{2 n}, b_{2 n}\right)$ is a linear 
extension of $\mathcal{W}_{G_{n, P}(i)}$, then one can define $F$ so that $F\left(a_{i}, b_{i}\right)=i$ and it will automatically be the case that $F \in \mathcal{M P}_{n}^{P^{(i)}}$.

We can define a similar poset for maximum packings of $\tau^{(i)}$ of length $2 n+1$. Note that in a maximum packing $F \in \mathcal{M P}_{n}^{P^{(i)}}$, the element $a$ in the top right-hand corner of $F$ corresponds to the last element of $\sigma(F)$ so that, to account for the last element in a permutation $\alpha=\alpha_{1} \ldots \alpha_{2 n+1} \in \mathcal{A}_{2 n+1}$ which has $\tau^{(i)}$-matches starting at positions $1,3, \ldots 2 n-3$, we must add an extra element $b$ to graph $G_{n, P^{(i)}}$ with a directed arrow from $b$ to $a$ since we know that $\alpha_{2 n}>\alpha_{2 n+1}$. We let $G_{n, P^{(i)}}^{+}$denote this extended graph. For example, the graph $G_{6, P^{(1)}}^{+}$is pictured in the third line of Figure 10. It follows that $\mathrm{mp}_{2 n+1, \tau^{(i)}}$ equals the number of linear extensions of $\mathcal{W}_{G_{n, P^{(i)}}}$.

First consider the problem of computing $\operatorname{mp}_{2 n+1}^{P^{(1)}}$ for $n \geqslant 2$. In this case, let $a$ be the rightmost element in the top row of $G_{n, P^{(1)}}$. Since there is a directed path in $G_{n, P^{(1)}}$ from every element other than $a$ to $a$, it must be the case that $a$ is the last element in any linear extension of $\mathcal{W}_{G_{n, P^{(1)}}}$ and, hence, in any $F \in \mathcal{M P}_{n}^{P^{(1)}}, F(a)=2 n$. Note that the same thing happens in $G_{n, P^{(1)}}^{+}$. That is, there is a directed path in $G_{n, P^{(1)}}^{+}$from every element other than $a$ to $a$. Thus it must be the case that $a$ is the last element in any linear extension of $\mathcal{W}_{G_{n, P}^{+}(1)}$ so that $a$ would be assigned the label $2 n+1$ in any linear extension. But then it is easy to see that $b$ can be assigned any element in $\{1, \ldots, 2 n\}$. Thus once we pick the value assigned to $b$, then the number of linear extensions of $G_{n, P^{(1)}}^{+}$just reduces to the number of linear extensions of $G_{n, P^{(1)}}$ which is $C_{n-1}$. Thus $\operatorname{mp}_{2 n+1, \tau^{(1)}}=2 n C_{n-1}$.

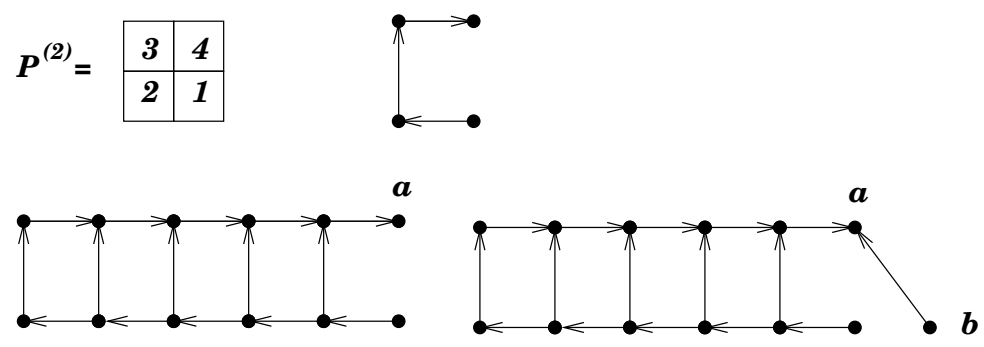

Figure 11: The graphs $G_{n, P^{(2)}}$ and $G_{n, P^{(2)}}^{+}$.

In Figure 11, we have pictured the graphs of $G_{6, P^{(2)}}$ and $G_{6, P^{(2)}}^{+}$in the second line. In this case, it is easy to see that there is a unique linear extension of $\mathcal{W}_{G_{n, P}(2)}$ and the rightmost top element $a$ must be the largest element $2 n$ since there is a directed path in $G_{n, P^{(2)}}$ from every element other than $a$ to $a$. The same thing happens in $G_{n, P^{(2)}}^{+}$, namely $2 n+1$ must be assigned to $a$ since there is a directed path in $G_{n, P^{(2)}}^{+}$from every element other than $a$ to $a$. But then it is easy to see that $b$ can be assigned to any element in $\{1, \ldots, 2 n\}$. Thus once we pick a value that is assigned to $b$, then the number of linear extensions of $G_{n, P^{(2)}}^{+}$just reduces to the number of linear extensions of $G_{n, P^{(2)}}$ which is just 1. Thus $\operatorname{mp}_{2 n+1, \tau^{(2)}}=2 n$.

In Figure 12, we have pictured the graphs of $G_{6, P^{(3)}}$ and $G_{6, P^{(3)}}^{+}$in the second line. Now 


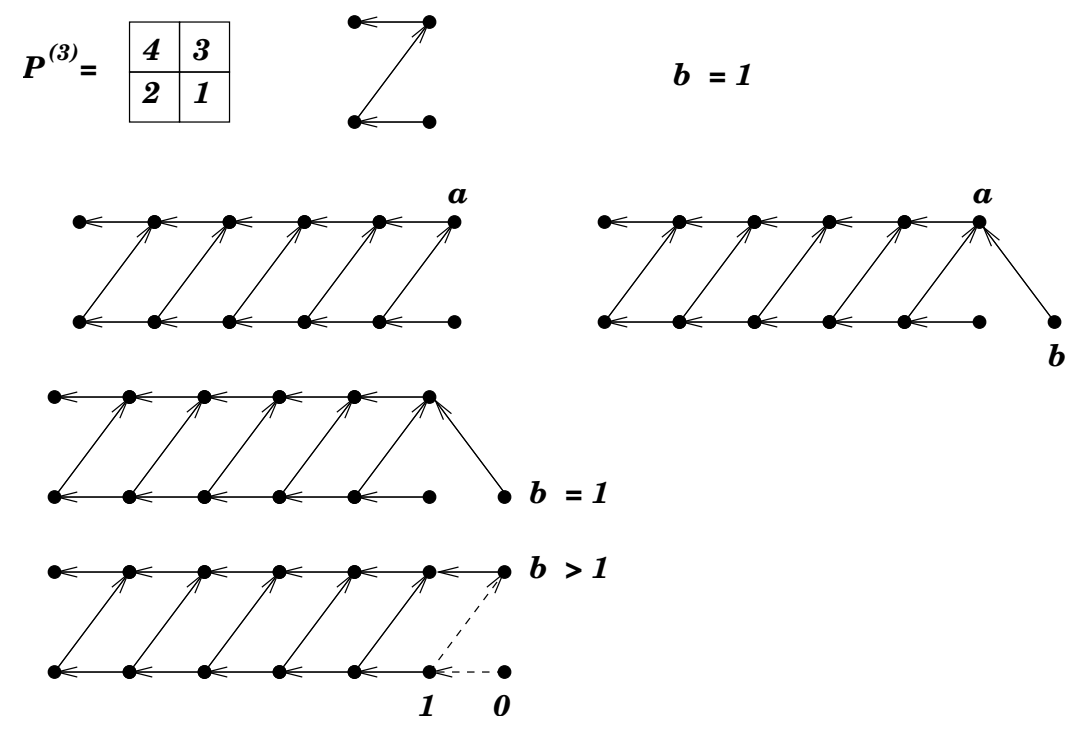

Figure 12: The graphs $G_{n, P^{(3)}}$ and $G_{n, P^{(3)}}^{+}$.

consider the element $b$ in $G_{n, P^{(3)}}^{+}$. If we assign $b$ the value 1 , then there is no restriction on the linear extensions of the remaining elements so that we get a total of $C_{n-1}$ linear extensions in that case since $\mathrm{mp}_{n, P^{(3)}}=C_{n-1}$. However, if $b>1$, then it is easy to see that the rightmost bottom element must be the first element in any linear extension since there is a directed path from that element to any other element which is not equal to $b$. Thus the rightmost bottom element must be assigned to 1 . It then follows that we can extend the graph $G_{n, P^{(3)}}^{+}$to a graph $G_{n, P^{(3)}}^{++}$by adding a new element 0 and adding new directed edges connecting 0 to 1 and 1 to $b$. This process is pictured on line 4 of Figure 12. It is easy to see that the number of linear extensions of $G_{n, P^{(3)}}^{+}$where $b>1$ is just the number of linear extensions of $G_{n, P^{(3)}}^{++}$which is the same as the number of linear extensions of $G_{n+1, P^{(3)}}$. Since the number of linear extensions of $G_{n+1, P^{(3)}}$ is $C_{n}$, it follows that $\mathrm{mp}_{2 n+1, \tau^{(3)}}=C_{n-1}+C_{n}$.

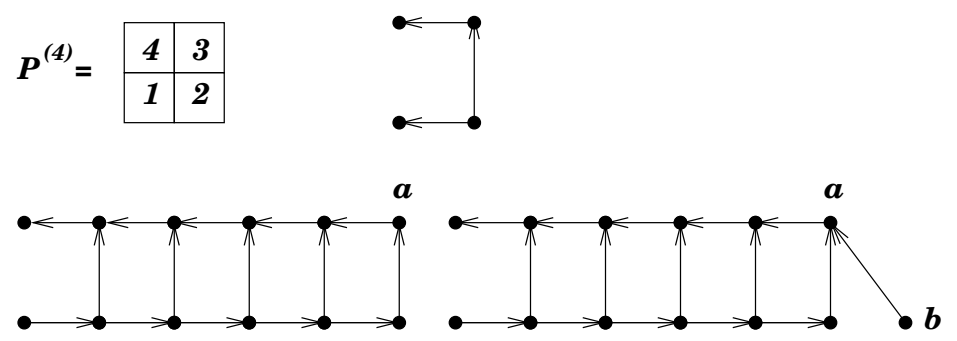

Figure 13: The graphs $G_{n, P^{(4)}}$ and $G_{n, P^{(4)}}^{+}$.

In Figure 13, we have pictured the graphs of $G_{6, P^{(4)}}$ and $G_{6, P^{(4)}}^{+}$in the second line. In this case, it is easy to see that there is a unique linear extension of $\mathcal{W}_{G_{n, P}^{(4)}}$ and the 
rightmost top element $a$ of $G_{n, P^{(4)}}$ must be the $(n+1)$ st element in the linear extension of $\mathcal{W}_{G_{6, P^{(4)}}}$ since there are $n$ elements $x$ for which there is a directed path in $\bar{G}_{n, P^{(4)}}$ from $x$ to $a$ and there are $n-1$ elements $y$ such that there is a directed path from $a$ to $y$ in $\bar{G}_{n, P^{(4)}}$. Similarly, $a$ must be the $(n+2)$ nd element in any linear extension of $\mathcal{W}_{G_{6, P^{(4)}}^{+}}$since there are $n+1$ elements $x$ for which there is a directed path in $\bar{G}_{n, P^{(4)}}^{+}$from $x$ to $a$ and there are $n-1$ elements $y$ such that there is a directed path from $a$ to $y$ in $\bar{G}_{n, P^{(4)}}^{+}$. Hence we can assign $b$ to be any element from $1, \ldots, n+1$. Once we pick a value for $b$, then the number of linear extensions of $\bar{G}_{n, P^{(4)}}^{+}$just reduces to the number of linear extensions of $\bar{G}_{n, P^{(4)}}$ which is just 1 . Thus $\operatorname{mp}_{2 n+1, \tau^{(4)}}=n+1$.

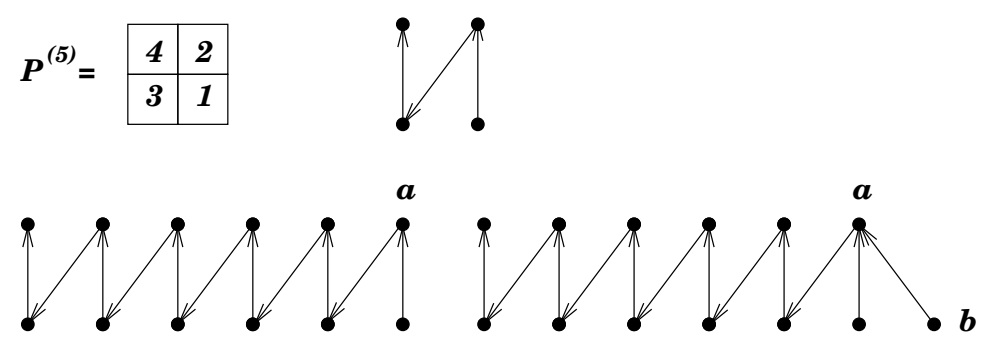

Figure 14: The graphs $G_{n, P^{(5)}}$ and $G_{n, P^{(5)}}^{+}$.

In Figure 14, we have pictured the graphs of $G_{6, P^{(5)}}$ and $G_{6, P^{(5)}}^{+}$in the second line. In the case of $G_{n, P^{(5)}}^{+}$, it is easy to see that the rightmost top element $a$ must be the third element in any linear extension of $\mathcal{W}_{G_{6, P^{(5)}}}$. Thus we have two linear extensions depending upon how we order the two elements that have a directed edge into $a$. Hence $\mathrm{mp}_{2 n+1, P^{(5)}}=2$.

\section{Computing $G M P_{n, \tau^{(i)}}(x)$}

In this section, we shall study the problem of computing $G M P_{n, \tau^{(i)}}(x)$ for $n \geqslant 1$ and $i=1, \ldots, 5$. First it is easy to see that for any $i, G M P_{1, \tau^{(i)}}(x)=G M P_{2, \tau^{(i)}}(x)=1$, $G M P_{3, \tau^{(i)}}(x)=-1$, and $G M P_{4, \tau^{(i)}}(x)=x-2$. That is, there is only one generalized maximum packing of length 1 which consists of a block of length 1 and weight 1 . Similarly, there is only one generalized maximum packing of length 2 which consists of a block of length 2 and weight 1 . There is only one generalized maximum packing of length 3 , namely, 123 where 12 is a block of length 2 and 3 is a block of length one. Thus $G M P_{3, \tau^{(i)}}=w(123)=-1$. There are two generalized maximum packings of length 4 , namely, 1234 which consists of two blocks of length 2 and has weight -1 and $\tau^{(i)}$ which consists of a single block with weight $x-1$. Thus $G M P_{4, \tau^{(i)}}=x-2$.

In general, we do not know how to find closed formulas for $G M P_{n, \tau^{(i)}}(x)$ as function of $n$, but for $i \in\{1,2,4\}$ there are simple recursions for computing $G M P_{n, \tau^{(i)}}(x)$. The key to our ability to develop recursions for $G M P_{n, \tau^{(i)}}(x)$ in the case where $i \in\{1,2,4\}$ is due 
to the fact that $\tau^{(1)}, \tau^{(2)}$, and $\tau^{(4)}$ either start with 1 or end with 4 . This will allow us to develop recursions based on either the length of the first block or the length of the last block in a generalized maximum packing. Neither $\tau^{(3)}=2413$ nor $\tau^{(5)}=3412$ start with 1 or end with 4 and we have not been able to find any simple recursions for $G M P_{n, \tau^{(3)}}$ or $G M P_{n, \tau^{(5)}}$.

The easiest case is for $\tau^{(1)}=1324$ where we have the following theorem.

Theorem 4. For $n \geqslant 3$,

$$
G M P_{2 n, \tau^{(1)}}(x)=C_{n-1}(x-1)^{n-1}-\sum_{k=1}^{n-1} C_{k-1}(x-1)^{k-1} G M P_{2 n-2 k, \tau^{(1)}}(x)
$$

and, for $n \geqslant 2, G M P_{2 n+1, \tau^{(1)}}(x)=-G M P_{2 n, \tau^{(1)}}(x)$.

Proof. It is easy to see from the form of the graphs $G_{2 n, P^{(1)}}$ that any maximum packing $\sigma \in \mathcal{M P}_{2 n, \tau^{(1)}}$ must start with 1 and end with $2 n$. By the definition of a generalized maximum packing whose block structure is $B_{1} \ldots B_{k}$, the last element of $B_{i}$ must be smaller than the first element of $B_{i+1}$ for all $i<k$. Thus all the elements of $B_{i}$ are smaller than any element in $B_{i+1}$ for all $i<k$.

Now suppose that $n \geqslant 3$ and $\sigma=\sigma_{1} \ldots \sigma_{2 n} \in \mathcal{G M P}_{2 n, \tau^{(1)}}$. There are two possibilities.

Case 1. $\sigma$ consists of a single block.

In this case $\sigma$ is a maximum packing of $\tau^{(1)}$ and $w(\sigma)=(x-1)^{n-1}$. Since $\operatorname{mp}_{2 n, \tau^{(i)}}=C_{n-1}$, the contribution of the permutations in case 1 to $G M P_{2 n, \tau^{(1)}}(x)$ is $C_{n-1}(x-1)^{n-1}$.

Case 2. $\sigma$ has block structure $B_{1} \ldots B_{s}$ where $s \geqslant 2$.

If $B_{1}$ is of length 2 , then $B_{1}=12$ and has weight $-1=-C_{0}$ and $\operatorname{red}\left(B_{2} \ldots B_{s}\right)$ is a generalized maximum packing for $\tau^{(1)}$ of length $2 n-2$. If $B_{1}$ has length $2 k$ where $k \geqslant 2$, then $B_{1}=1 \ldots 2 k$ is a maximum packing for $\tau^{(1)}$ of length $2 k$ which has weight $-(x-1)^{k-1}$ and $\operatorname{red}\left(B_{2} \ldots B_{s}\right)$ is a generalized maximum packing of length $2 n-2 k$. Then there are $C_{k-1}$ choices for $B_{1}$. Hence the contribution of the permutations in case 2 to $G M P_{2 n, \tau^{(1)}}(x)$ is $-\sum_{k=1}^{n-1} C_{k-1}(x-1)^{k-1} G M P_{2 n-2 k, \tau^{(1)}}(x)$.

Thus for $n \geqslant 3$, (28) holds.

It is also easy to compute $G M P_{2 n+1, \tau^{(1)}}(x)$. That is, since a generalized maximum packing $\sigma \in \mathcal{A}_{2 n+1}$ has block structure $B_{1} \ldots B_{k}$ where $B_{k}$ has length 1 and $B_{1} \ldots B_{k-1}$ reduces to a generalized maximum packing for $\tau^{(1)}$ of length $2 n$, we know that the last element of $B_{k-1}$ is the largest element in $B_{1} \ldots B_{k-1}$ and hence the element in $B_{k}$ must be $2 n+1$. Thus in this case $G M P_{2 n+1, \tau^{(1)}}(x)=-G M P_{2 n, \tau^{(1)}}(x)$.

Here is the list of the first few values of $G M P_{2 n, \tau^{(1)}}(x)$.

$$
\begin{aligned}
& \operatorname{GMP}_{2, \tau^{(1)}}(x)=1 \\
& \operatorname{GMP}_{4, \tau^{(1)}}(x)=-2+x
\end{aligned}
$$




$$
\begin{aligned}
G M P_{6, \tau^{(1)}}(x)= & 5-6 x+2 x^{2} \\
G M P_{8, \tau^{(1)}}(x)= & -14+28 x-20 x^{2}+5 x^{3} \\
G M P_{10, \tau^{(1)}}(x)= & 42-120 x+135 x^{2}-70 x^{3}+14 x^{4} \\
G M P_{12, \tau^{(1)}}(x)= & -132+495 x-770 x^{2}+616 x^{3}-252 x^{4}+42 x^{5} \\
G M P_{14, \tau^{(1)}}(x)= & 429-2002 x+4004 x^{2}-4368 x^{3}+2730 x^{4}-924 x^{5}+132 x^{6} \\
G M P_{16, \tau^{(1)}}(x)= & -1430+8008 x-19656 x^{2}+27300 x^{3}-23100 x^{4}+ \\
& 11880 x^{5}-3432 x^{6}+429 x^{7}
\end{aligned}
$$

Plugging these values into the generating functions (6) and (7), we have computed the following table of values of $A_{n, \tau^{(1)}}(x)=\sum_{\sigma \in \mathcal{A}_{n}} x^{\tau^{(1)}-\operatorname{mch}(\sigma)}$.

$$
\begin{aligned}
& A_{1, \tau^{(1)}}=1 \\
& A_{2, \tau^{(1)}}=1 \\
& A_{3, \tau^{(1)}}=2 \\
& A_{4, \tau^{(1)}}=4+x \\
& A_{5, \tau^{(1)}}=12+4 x \\
& A_{6, \tau^{(1)}}=35+24 x+2 x^{2} \\
& A_{7, \tau^{(1)}}=142+118 x+12 x^{2} \\
& A_{8, \tau^{(1)}}=546+672 x+162 x^{2}+5 x^{3} \\
& A_{9, \tau^{(1)}}=2816+3968 x+1112 x^{2}+40 x^{3} \\
& A_{10, \tau^{(1)}}=13482+24660 x+11145 x^{2}+1220 x^{3}+14 x^{4} \\
& A_{11, \tau^{(1)}}=84764+170996 x+87200 x^{2}+10666 x^{3}+168 x^{4}
\end{aligned}
$$

In this case, one can explicitly calculate the values of $\left.G M P_{n, \tau^{(1)}}(x)\right|_{x^{0}}=G M P_{n, \tau^{(1)}}(0)$ and $\left.G M P_{n, \tau^{(1)}}(x)\right|_{x}$. One can obviously get recursions for $\left.G M P_{n, \tau^{(1)}}(x)\right|_{x^{k}}$ for $k \geqslant 2$ but we could not find nice explicit formulas for such coefficients. For example, the sequence of absolute values of the coefficients of $\left.G M P_{n, \tau^{(1)}}(x)\right|_{x^{2}}$ does not appear in the On-Line Encyclopedia of Integer Sequences (OEIS).

Theorem 5. 1. For all $m \geqslant 1, G_{2 m, \tau^{(1)}}(0)=(-1)^{m-1} C_{m}$ and $G_{2 m+1, \tau^{(1)}}(0)=(-1)^{m} C_{m}$.

2. For all $m \geqslant 2,\left.G_{2 m, \tau^{(1)}}(x)\right|_{x}=(-1)^{m}\left(\begin{array}{c}2 m \\ m-2\end{array}\right)$ and $\left.G_{2 m+1, \tau^{(1)}}(x)\right|_{x}=(-1)^{m+1}\left(\begin{array}{c}2 m \\ m-2\end{array}\right)$.

Proof. For (1), our formula obviously holds for $m=1$ and $m=2$. Now if $n>2$ and we assume that $G_{2 m, \tau^{(1)}}(0)=(-1)^{m-1} C_{m}$ for $m<n$, then by (28), we have

$$
\begin{aligned}
G M P_{2 n, \tau^{(1)}}(0) & =C_{n-1}(-1)^{n-1}-\sum_{k=1}^{n-1} C_{k-1}(-1)^{k-1} G M P_{2 n-2 k, \tau^{(i)}}(0) \\
& =C_{n-1}(-1)^{n-1}-\sum_{k=1}^{n-1} C_{k-1}(-1)^{k-1}(-1)^{n-k-1} C_{n-k}
\end{aligned}
$$




$$
=(-1)^{n-1} \sum_{k=1}^{n} C_{k-1} C_{n-k}=(-1)^{n-1} C_{n} .
$$

For $(2),\left.G M P_{4, \tau^{(1)}}(x)\right|_{x}=1$ so our formula holds for $n=2$. For $n>2$, we have that

$$
\begin{aligned}
\left.G M P_{2 n, \tau^{(1)}}(x)\right|_{x}= & \left.C_{n-1}(x-1)^{n-1}\right|_{x}-\left.\left.\sum_{k=1}^{n-1}\left(C_{k}(x-1)^{k} \mid\right)\right|_{x}\left(G M P_{2 n-2 k-2, \tau^{(1)}}(x)\right)\right|_{x^{0}} \\
& -\left.\left.\sum_{k=1}^{n-1}\left(C_{k}(x-1)^{k}\right)\right|_{x^{0}}\left(G M P_{2 n-2 k-2, \tau^{(1)}}(x)\right)\right|_{x} .
\end{aligned}
$$

Using induction and some simplifications with binomial coefficients, one can show that this is equivalent to the following identity:

$$
\begin{aligned}
\left.(-1)^{n} G M P_{2 n, \tau^{(1)}}(x)\right|_{x}= & \left(\begin{array}{c}
2 n-1 \\
n-2
\end{array}\right)+\sum_{k=1}^{n-2} \frac{1}{n-k}\left(\begin{array}{c}
2 k \\
k-1
\end{array}\right)\left(\begin{array}{c}
2 n-2 k-2 \\
n-k-1
\end{array}\right) \\
& +\sum_{k=1}^{n-3} \frac{1}{k+1}\left(\begin{array}{c}
2 k \\
k
\end{array}\right)\left(\begin{array}{c}
2 n-2 k-2 \\
n-k-3
\end{array}\right)
\end{aligned}
$$

One can then verify that the right-hand side of (29) is equal to $\left(\begin{array}{c}2 n \\ n-2\end{array}\right)$ by induction using a series of routine manipulations and some simple identities for binomial coefficients.

Let $N_{n, \tau^{(1)}}$ denote the number of permutations $\sigma \in \mathcal{A}_{n}$ such that $\tau^{(1)}-\operatorname{mch}(\sigma)=0$ and $U_{n, \tau^{(1)}}$ denote the number of permutations $\sigma \in \mathcal{A}_{n}$ such that $\tau^{(1)}-\operatorname{mch}(\sigma)=1$. Our previous theorem allows us to compute explicit generating functions for $N_{n, \tau^{(1)}}$ and $U_{n, \tau^{(1)}}$.

Theorem 6. Let $R(t)=\sum_{n \geqslant 1}(-1)^{n-1} C_{n} \frac{t^{2 n}}{(2 n) !}$ and $S(t)=\sum_{n \geqslant 2}(-1)^{n}\left(\begin{array}{c}2 n \\ n-2\end{array}\right) \frac{t^{2 n}}{(2 n) !}$. Then

$$
\begin{aligned}
& 1+\sum_{n \geqslant 1} N_{2 n, \tau^{(1)}} \frac{t^{2 n}}{(2 n) !}=\frac{1}{1-R(t)}, \\
& \sum_{n \geqslant 0} N_{2 n+1, \tau^{(1)}} \frac{t^{2 n+1}}{(2 n+1) !}=\frac{t-\int_{0}^{t} R(z) d z}{1-R(t)}, \\
& 1+\sum_{n \geqslant 1} U_{2 n, \tau^{(1)}} \frac{t^{2 n}}{(2 n) !}=\frac{S(t)}{(1-R(t))^{2}}, \text { and } \\
& \sum_{n \geqslant 0} N_{2 n+1, \tau^{(1)}} \frac{t^{2 n+1}}{(2 n+1) !}=\frac{(1-R(t))\left(\int_{0}^{t} S(z) d z\right)+S(t)\left(t-\int_{0}^{t} R(z) d z\right)}{(1-R(t))^{2}} .
\end{aligned}
$$

Proof. By Theorem 5,

$$
\sum_{n \geqslant 1} G M P_{2 n, \tau^{(1)}}(x) \frac{t^{2 n}}{(2 n) !}=R(t)+x S(t)+O\left(x^{2}\right)
$$


and

$$
\begin{aligned}
\sum_{n \geqslant 1} G M P_{2 n-1, \tau^{(1)}}(x) \frac{t^{2 n-1}}{(2 n-1) !} & =t+\sum_{n \geqslant 2} G M P_{2 n-1, \tau^{(1)}}(x) \frac{t^{2 n-1}}{(2 n-1) !} \\
& =t-\sum_{n \geqslant 2} G M P_{2 n-2, \tau^{(1)}}(x) \frac{t^{2 n-1}}{(2 n-1) !} \\
& =t-\int_{0}^{t} R(z)+x S(z)+O\left(x^{2}\right) d z .
\end{aligned}
$$

Plugging these expressions into Theorem 1, we see that

$$
A_{\tau^{(1)}}(t, x)=\frac{1}{1-R(t)-x S(t)+O\left(x^{2}\right)}
$$

and

$$
B_{\tau^{(1)}}(t, x)=\frac{t-\int_{0}^{t} R(z) d z-x \int_{0}^{t} S(z) d z+O\left(x^{2}\right)}{1-R(t)-x S(t)+O\left(x^{2}\right)} .
$$

Equations (30) and (31) follow by putting $x=0$ in (35) and (36), respectively.

To prove (32) and (33), note that

$$
\begin{aligned}
\left.A_{\tau^{(1)}}(t, x)\right|_{x} & =\left.\left(1+\sum_{n \geqslant 1}(R(t)+x S(t))^{n}\right)\right|_{x}=\sum_{n \geqslant 1} n S(t)(R(t))^{n-1} \\
& =\frac{S(t)}{(1-R(t))^{2}} .
\end{aligned}
$$

Similarly, one can compute that

$$
\begin{aligned}
\left.B_{\tau^{(1)}}(t, x)\right|_{x} & =\frac{\left(t-\int_{0}^{t} R(z) d z\right) S(t)}{(1-R(t))^{2}}+\frac{\int_{0}^{t} S(z) d z}{1-R(t)} \\
& =\frac{(1-R(t))\left(\int_{0}^{t} S(z) d z\right)+S(t)\left(t-\int_{0}^{t} R(z) d z\right)}{(1-R(t))^{2}} .
\end{aligned}
$$

Unfortunately, we have not been able to prove similar results for $\tau^{(i)}$ where $i \geqslant 2$ because in these cases, we have not been able to find explicit formulas for $G M P_{n, \tau^{(i)}}(0)$ or $\left.G M P_{n, \tau^{(i)}}(x)\right|_{x}$.

Next we consider recursions for $G M P_{n, \tau^{(2)}}(x)$.

Theorem 7. For $n \geqslant 3$,

$$
G M P_{2 n, \tau^{(2)}}(x)=(x-1)^{n-1}-\sum_{j=1}^{n-1}\left(\begin{array}{c}
2 n-j-1 \\
j-1
\end{array}\right)(x-1)^{j-1} G M P_{2 n-2 j, \tau^{(2)}}(x)
$$

and, for $n \geqslant 2, G M P_{2 n+1, \tau^{(2)}}(x)=-G M P_{2 n, \tau^{(2)}}(x)$. 
Proof. It is clear from the graph $G_{n, P^{(2)}}$ that in the unique maximum packing $\sigma=$ $\sigma_{1} \ldots \sigma_{2 n} \in \mathcal{A}_{2 n}$ for $\tau^{(2)}, \sigma_{2} \sigma_{4} \ldots \sigma_{2 n}=(n+1)(n+2) \ldots(2 n)$ and $\sigma_{1} \sigma_{3} \ldots \sigma_{n}=n(n-$ 1) $(n-2) \ldots 1$. It follows that in a generalized maximum packing $\alpha \in S_{2 n}$ with block structure $B_{1} \ldots B_{k}$, the last element of each block $B_{i}$ is the largest element in the block.

If $\sigma$ is a maximum packing for $\tau^{(2)}$ with block structure $B_{1} \ldots B_{k}$, we shall simply write $\sigma=B_{1} \ldots B_{k}$. Our recursion follows by classifying the generalized packings $\sigma=B_{1} B_{2} \ldots B_{k}$ for $\tau^{(2)}$ by the size of the last block $B_{k}$. If $k=1$, then $\sigma$ is unique and $w(\sigma)=(x-1)^{n-1}$. Thus suppose that $k \geqslant 2$. If $B_{k}$ is length two, then its two elements must be $(2 n-1)$ and $(2 n)$ since they must be larger than all the largest elements in each block $B_{i}$ for $i \neq k$. In that case, $B_{1} \ldots B_{k-1}$ is just a generalized maximum packing for $\tau^{(2)}$ of length $2 n-2$ and $w(\sigma)=-w\left(B_{1} \ldots B_{k-1}\right)$. Thus such permutations contribute $-G M P_{2 n-2, \tau^{(2)}}(x)$ to $G M P_{2 n, \tau^{(2)}}(x)$. If $B_{k}$ has length $2 j$ where $j \geqslant 2$, then $B_{k}=\sigma_{2 n-2 j+1} \ldots \sigma_{2 n}$ reduces to a maximum packing for $\tau^{(2)}$ of length $2 j$ and $w(\sigma)=-(x-1)^{j-1} w\left(B_{1} \ldots B_{k-1}\right)$. Then we know that $\sigma_{2 n-2 j+1}<\sigma_{2 n-2 j+2}<\sigma_{2 n-2 j+4}<$ $\cdots<\sigma_{2 n}$ must be the $j+1$ largest elements from $\{1, \ldots, 2 n\}$ since they will be larger than all the remaining elements of $B_{k}$ and larger than the largest element of $B_{i}$ for $i \neq k$. It follows that first element of block $B_{k}$ is $(2 n-j)$. Our conditions for a generalized maximum packing for $\tau^{(2)}$ do not impose any relations between the remaining elements of $B_{k}$, namely $\sigma_{2 n-2 j+3}, \sigma_{2 n-2 j+5}, \ldots, \sigma_{2 n-1}$, and the elements in blocks $B_{1}, \ldots, B_{k-1}$. Thus we have $\left(\begin{array}{c}2 n-j-1 \\ j-1\end{array}\right)$ ways to choose those elements. Once we have chosen those elements, then $B_{1} \ldots B_{k-1}$ must reduce to a generalized maximum packing for $\tau^{(2)}$ of length $2 n-2 j$. Thus such permutations contribute $-\left(\begin{array}{c}2 n-j-1 \\ j-1\end{array}\right)(x-1)^{j-1} G M P_{2 n-2 j, \tau^{(2)}}(x)$ to $G M P_{2 n, \tau^{(2)}}(x)$. Hence (39) holds.

Again, it is easy to compute $G M P_{2 n+1, \tau^{(2)}}(x)$. That is, since a generalized maximum packing $\sigma \in \mathcal{A}_{2 n+1}$ has block structure $B_{1} \ldots B_{k}$ where $B_{k}$ has length 1 and $B_{1} \ldots B_{k-1}$ reduces to a generalized maximum packing for $\tau^{(2)}$ of length $2 n$, we know that the last element of $B_{k-1}$ is the largest element in $B_{1} \ldots B_{k-1}$ and hence the element in $B_{k}$ must be $2 n+1$. Thus in this case $G M P_{2 n+1, \tau^{(2)}}(x)=-G M P_{2 n, \tau^{(2)}}(x)$.

Here is the list of the first few values of $G M P_{2 n, \tau^{(1)}}(x)$.

$$
\begin{aligned}
& \operatorname{GMP}_{2, \tau^{(2)}}(x)= \\
& \operatorname{GMP}_{4, \tau^{(2)}}(x)= x-2 \\
& \operatorname{GMP}_{6, \tau^{(2)}}(x)= 6-6 x+x^{2} \\
& \operatorname{GMP}_{8, \tau^{(2)}}(x)=-23+36 x-15 x^{2}+x^{3} \\
& G M P_{10, \tau^{(2)}}(x)= 106-229 x+160 x^{2}-37 x^{3}+x^{4} \\
& G M P_{12, \tau^{(2)}}(x)=-567+1574 x-1566 x^{2}+650 x^{3}-93 x^{4}+x^{5} \\
& G M P_{14, \tau^{(2)}}(x)= 3434-11706 x+15248 x^{2}-9310 x^{3}+2572 x^{4}-238 x^{5}+x^{6} \\
& G M P_{16, \tau^{(2)}}(x)=-23137+93831 x-151933 x^{2}+123814 x^{3}-52136 x^{4}+ \\
& 10175 x^{5}-616 x^{6}+x^{7}
\end{aligned}
$$

In this case the sequence $\left((-1)^{n-1} G M P_{2 n, \tau^{(2)}}(0)\right)_{n \geqslant 1}$ which starts out with $1,2,6,23,106,567,23137, \ldots$ is sequence A125273 in the OEIS. Unfortunately, there seems 
to be no exact formula for this sequence. The sequence $\left(\left.(-1)^{n} G M P_{2 n, \tau^{(2)}}(x)\right|_{x}\right)_{n \geqslant 1}$ which starts out $1,6,36,1574,11706,933831, \ldots$ does not appear in the OEIS.

Plugging these values into the generating functions (6) and (7), we have computed the following table of values of $A_{n, \tau^{(2)}}(x)=\sum_{\sigma \in \mathcal{A}_{n}} x^{\tau^{(2)}-\operatorname{mch}(\sigma)}$.

$$
\begin{aligned}
A_{1, \tau^{(2)}} & =1 \\
A_{2, \tau^{(2)}} & =1 \\
A_{3, \tau^{(2)}} & =2 \\
A_{4, \tau^{(2)}} & =4+x \\
A_{5, \tau^{(2)}} & =12+4 x \\
A_{6, \tau^{(2)}} & =36+24 x+x^{2} \\
A_{7, \tau^{(2)}} & =148+118 x+6 x^{2} \\
A_{8, \tau^{(2)}} & =593+680 x+111 x^{2}+x^{3} \\
A_{9, \tau^{(2)}} & =3128+4032 x+768 x^{2}+8 x^{3} \\
A_{10, \tau^{(2)}} & =15676+25691 x+8680 x^{2}+473 x^{3}+x^{4} \\
A_{11, \tau^{(2)}} & =101094+180134 x+68326 x^{2}+4228 x^{3}+10 x^{4}
\end{aligned}
$$

Next we consider recursions for $G M P_{2 n, \tau^{(4)}}(x)$.

Theorem 8. For $n \geqslant 3$,

$$
G M P_{2 n, \tau^{(4)}}(x)=(x-1)^{n-1}-\sum_{j=1}^{n-1}\left(\begin{array}{c}
2 n-j-1 \\
j-1
\end{array}\right) G M P_{2 n-2 j, \tau^{(4)}}(x)
$$

and, for $n \geqslant 2$,

$$
G M P_{2 n+1, \tau^{(4)}}(x)=-n(x-1)^{n-1}-\sum_{j=1}^{n-1}\left(\begin{array}{c}
2 n-j \\
j-1
\end{array}\right) G M P_{2 n+1-2 j, \tau^{(4)}}(x) .
$$

Proof. It is clear from the graph $G_{n, P^{(4)}}$ that in the unique maximum packing $\sigma=$ $\sigma_{1} \ldots \sigma_{2 n} \in \mathcal{A}_{2 n}$ for $\tau^{(4)}, \sigma_{2} \sigma_{4} \ldots \sigma_{2 n}=(2 n)(2 n-1) \ldots(n+1)$ and $\sigma_{1} \sigma_{3} \ldots \sigma_{2 n-1}=12 \ldots n$. It follows that in a generalized maximum packing $\alpha \in S_{2 n}$ with block structure $B_{1} \ldots B_{k}$, the first element of each block $B_{i}$ is the smallest element in the block.

If $\sigma$ is a maximum packing for $\tau^{(4)}$ with block structure $B_{1} \ldots B_{k}$, we shall simply write $\sigma=B_{1} \ldots B_{k}$. We will classify the generalized maximum packings $\sigma=B_{1} \ldots B_{k}$ of $\tau^{(4)}$ of length $2 n$ by the size of the first block $B_{1}$. If $k=1$, then $\sigma$ is unique and $w(\sigma)=(x-1)^{n-1}$. Next consider the case where $k \geqslant 2$. If $B_{1}$ is length two then, its two elements must be 1 and 2 since they must be smaller than all the smallest elements in each block $B_{i}$ for $i>1$. In that case $B_{2} \ldots B_{k}$ is just a generalized maximum packing for $\tau^{(4)}$ of length $2 n-2$ and $w(\sigma)=-w\left(B_{2} \ldots B_{k}\right)$. Thus such permutations contribute $-G M P_{2 n-2, \tau^{(4)}}(x)$ to $G M P_{2 n, \tau^{(4)}}(x)$. If $B_{1}$ is of size $2 j$ where $j \geqslant 2$, then $B_{1}=\sigma_{1} \ldots \sigma_{2 j}$ reduces to a maximum packing for $\tau^{(4)}$ of length $2 j$ where $2 \leqslant j \leqslant n-1$ and $w(\sigma)=$ 
$-(x-1)^{j-1} w\left(B_{2} \ldots B_{k}\right)$. Then it must be the case $\sigma_{1}<\sigma_{3}<\sigma_{5}<\cdots<\sigma_{2 j-1}<\sigma_{2 j}$ must be the $j+1$ smallest elements from $\{1, \ldots, 2 n\}$ since they will be smaller than all the remaining elements of $B_{1}$ and smaller than the smallest element of $B_{i}$ for $i>1$. It follows that the last element of block $B_{1}$ is $(j+1)$. Our definitions for a generalized maximum packing for $\tau^{(4)}$ do not impose any relations between the remaining elements of $B_{1}$, namely $\sigma_{2}, \sigma_{4}, \ldots, \sigma_{2 j-2}$, and the elements in blocks $B_{2}, \ldots, B_{k}$. Thus we have $\left(\begin{array}{c}2 n-j-1 \\ j-1\end{array}\right)$ ways to choose those elements. Once we have chosen those elements, then $B_{2} \ldots B_{k}$ must reduce to a generalized maximum packing for $\tau^{(4)}$ of length $2 n-2 j$. Thus such permutations contribute $-\left(\begin{array}{c}2 n-j-1 \\ j-1\end{array}\right)(x-1)^{j-1} G M P_{2 n-2 j, \tau^{(4)}}(x)$ to $G M P_{2 n, \tau^{(4)}}(x)$. Hence $(40)$ holds.

This is the same recursion as (39) and it implies that $A_{\tau^{(2)}}(t, x)=A_{\tau^{(4)}}(t, x)$. This is no surprise since even length up-down permutations are closed under reverse complement. That is, if $\sigma=\sigma_{1} \ldots \sigma_{n} \in S_{n}$, then the reverse of $\sigma, \sigma^{r}$, is defined to be $\sigma^{r}=\sigma_{n} \ldots \sigma_{1}$ and the complement of $\sigma, \sigma^{c}$, is defined by $\sigma^{c}=\left(n+1-\sigma_{1}\right) \ldots\left(n+1-\sigma_{n}\right)$. The reversecomplement of $\sigma$ is $\left(\sigma^{r}\right)^{c}$. It is easy to check that $\sigma \in \mathcal{A}_{2 n}$ if and only if $\left(\sigma^{r}\right)^{c} \in \mathcal{A}_{2 n}$ and that $\left(2314^{r}\right)^{c}=1423$. Thus the map which sends $\sigma \in \mathcal{A}_{2 n}$ to $\left(\sigma^{r}\right)^{c}$ shows that $A_{\tau^{(2)}}(t, x)=A_{\tau^{(4)}}(t, x)$.

It is not the case that $B_{\tau^{(2)}}(t, x)=B_{\tau^{(4)}}(t, x)$ since for $n \geqslant 2$, the number of maximum packings for $\tau^{(2)}$ of length $2 n+1$ is $2 n$ while the number of maximum packings for $\tau^{(4)}$ of length $2 n+1$ is $(n+1)$. Nevertheless, we can still develop a recursion for $G M P_{2 n+1, \tau^{(4)}}(x)$ for $n \geqslant 2$. That is, since any generalized maximum packing for $\tau^{(4)}$ of length $2 n+1$ has block structure $B_{1} \ldots B_{k}$ where $B_{k}$ has length 1 and $B_{1} \ldots B_{k-1}$ reduces to a generalized maximum packing for $\tau^{(4)}$ of length $2 n$, it will still be the case that the first element in each block is the smallest element.

Again, we will classify the generalized maximum packings $\sigma=B_{1} \ldots B_{k}$ of $\tau^{(4)}$ of length $2 n+1$ by the size of the first block $B_{1}$. If $k=2$, then $B_{1}$ has length $2 n$ and $B_{2}$ has length 1 . One can see by the graph of $G_{n, P^{(4)}}$ and the fact that $\sigma_{2 n}<\sigma_{2 n+1}$ that $\sigma_{2 n}=n+1$. Then we have $n$ choices for $\sigma_{2 n+1}$ and after we pick $\sigma_{2 n+1}, \sigma_{1} \ldots \sigma_{2 n}$ must reduce to a maximum packing of $\tau^{(4)}$. Since there is only one maximum packing of length $2 n$ for $\tau^{(4)}$, we have exactly $n$ such permutations and the weight of each such permutation is $-(x-1)^{n-1}$. Thus such permutations contribute $-n(x-1)^{n-1}$ to $G M P_{2 n+1, \tau^{(4)}}(x)$. Next consider the case where $k \geqslant 3$. If $B_{1}$ is length two, then its two elements must be 1 and 2 since they must be smaller than all the smallest elements in each block $B_{i}$ for $i>1$. In that case $B_{2} \ldots B_{k}$ is just a generalized maximum packing for $\tau^{(4)}$ of length $2 n-1$ and $w(\sigma)=$ $-w\left(B_{2} \ldots B_{k}\right)$. Thus such permutations contribute $-G M P_{2 n-1, \tau^{(4)}}(x)$ to $G M P_{2 n+1, \tau^{(4)}}(x)$. If $B_{1}$ is of size $2 j$ where $j \geqslant 2$, then $B_{1}=\sigma_{1} \ldots \sigma_{2 j}$ reduces to a maximum packing for $\tau^{(4)}$ of length $2 j$ where $2 \leqslant j \leqslant n-1$ and $w(\sigma)=-(x-1)^{j-1} w\left(B_{2} \ldots B_{k}\right)$. Then it must be the case $\sigma_{1}<\sigma_{3}<\sigma_{5}<\cdots<\sigma_{2 j-1}<\sigma_{2 j}$ must be the $j+1$ smallest elements from $\{1, \ldots, 2 n\}$ since they will be smaller than all the remaining elements of $B_{1}$ and smaller than the smallest element of $B_{i}$ for $i>1$. It follows that the last element of block $B_{1}$ is $(j+1)$. Our definitions for a generalized maximum packing for $\tau^{(4)}$ do not impose any relations between the remaining elements of $B_{1}$, namely $\sigma_{2}, \sigma_{4}, \ldots, \sigma_{2 j-2}$, and the elements in blocks $B_{2}, \ldots, B_{k}$. Thus we have $\left(\begin{array}{c}2 n+1-j-1 \\ j-1\end{array}\right)$ ways to choose those elements. Once we have chosen those elements, then $B_{2} \ldots B_{k}$ must reduce to a generalized 
maximum packing for $\tau^{(4)}$ of length $2 n+1-2 j$. Thus such permutations contribute $-\left(\begin{array}{c}2 n-j \\ j-1\end{array}\right)(x-1)^{j-1} G M P_{2 n+1-2 j, \tau^{(4)}}(x)$ to $G M P_{2 n, \tau^{(4)}}(x)$. Hence (41) holds.

Here is the list of the first few values of $G M P_{2 n+1, \tau^{(4)}}(x)$.

$$
\begin{aligned}
G M P_{1, \tau^{(4)}}(x) & =1 \\
G M P_{3, \tau^{(4)}}(x) & =-1 \\
G M P_{5, \tau^{(4)}}(x) & =3-2 x \\
G M P_{7, \tau^{(4)}}(x) & =-10+12 x-3 x^{2} \\
G M P_{9, \tau^{(4)}}(x) & =42-74 x+37 x^{2}-4 x^{3} \\
G M P_{11, \tau^{(4)}}(x) & =-210+498 x-394 x^{2}+110 x^{3}-5 x^{4} \\
G M P_{13, \tau^{(4)}}(x) & =1199-3596 x+3946 x^{2}-1872 x^{3}+330 x^{4}-6 x^{5} \\
G M P_{15, \tau^{(4)}}(x) & =-7670+27908 x-39356 x^{2}+26604 x^{3}-8476 x^{4}+996 x^{5}-7 x^{6}
\end{aligned}
$$

In this case, the sequence $\left\{G M P_{2 n+1, \tau^{(4)}}(0)\right\}_{n \geqslant 0}$ is A125274 in the OEIS. Unfortunately, there is no exact formula for the elements in this sequence.

Plugging these values into the generating functions (6) and (7), we have computed the following table of values of $A_{n, \tau^{(4)}}(x)=\sum_{\sigma \in \mathcal{A}_{n}} x^{\tau^{(4)}-\operatorname{mch}(\sigma)}$.

$$
\begin{aligned}
& A_{1, \tau^{(4)}}=1 \\
& A_{2, \tau^{(4)}}=1 \\
& A_{3, \tau^{(4)}}=2 \\
& A_{4, \tau^{(4)}}=4+x \\
& A_{5, \tau^{(4)}}=13+3 x \\
& A_{6, \tau^{(4)}}=36+24 x+x^{2} \\
& A_{7, \tau^{(4)}}=165+103 x+4 x^{2} \\
& A_{8, \tau^{(4)}}=593+680 x+111 x^{2}+x^{3} \\
& A_{9, \tau^{(4)}}=3507+3832 x+592 x^{2}+5 x^{3} \\
& A_{10, \tau^{(4)}}=15676+25691 x+8680 x^{2}+473 x^{3}+x^{4} \\
& A_{11, \tau^{(4)}}=113387+179369 x+58016 x^{2}+3014 x^{3}+6 x^{4}
\end{aligned}
$$

As we mentioned in the introduction to this section, we have not be able to find simple recursions for $G M P_{n, \tau^{(3)}}(x)$ or $G M P_{n, \tau^{(5)}}(x)$. However, J. Harmse [12] computed the following initial values of $G M P_{n, \tau^{(3)}}$ and $G M P_{n, \tau^{(5)}}$ by computing the number of linear extensions of the posets associated with the various block structures of generalized maximal packings.

Here is the list of the first few values of $G M P_{2 n, \tau^{(3)}}(x)$.

$$
\begin{aligned}
& \operatorname{GMP}_{1, \tau^{(3)}}(x)=1 \\
& \operatorname{GMP}_{2, \tau^{(3)}}(x)=1 \\
& \operatorname{GMP}_{3, \tau^{(3)}}(x)=-1
\end{aligned}
$$




$$
\begin{aligned}
\operatorname{GMP}_{4, \tau^{(3)}}(x) & =-2+x \\
\operatorname{GMP}_{5, \tau^{(3)}}(x) & =3-2 x \\
G M P_{6, \tau^{(3)}}(x) & =9-10 x+2 x^{2} \\
G M P_{7, \tau^{(3)}}(x) & =-18+24 x-7 x^{2} \\
G M P_{8, \tau^{(3)}}(x) & =-74+132 x-64 x^{2}+5 x^{3} \\
G M P_{9, \tau^{(3)}}(x) & =190-376 x+213 x^{2}-26 x^{3} \\
G M P_{10, \tau^{(3)}}(x) & =974-2394 x+1927 x^{2}-520 x^{3}+14 x^{4} \\
G M P_{11, \tau^{(3)}}(x) & =-3078+8180 x-7287 x^{2}+2282 x^{3}-98 x^{4} \\
G M P_{12, \tau^{(3)}}(x) & =-17688+54228 x-59393 x^{2}+26807 x^{3}-3997 x^{4}+42 x^{5}
\end{aligned}
$$

Plugging these values into the generating functions (6) and (7), we have computed the following table of values of $A_{n, \tau^{(3)}}(x)=\sum_{\sigma \in \mathcal{A}_{n}} x^{\tau^{(3)}-\operatorname{mch}(\sigma)}$.

$$
\begin{aligned}
A_{1, \tau^{(3)}} & =1 \\
A_{2, \tau^{(3)}} & =1 \\
A_{3, \tau^{(3)}} & =2 \\
A_{4, \tau^{(3)}} & =4+x \\
A_{5, \tau^{(3)}} & =13+3 x \\
A_{6, \tau^{(3)}} & =39+20 x+2 x^{2} \\
A_{7, \tau^{(3)}} & =178+87 x+7 x^{2} \\
A_{8, \tau^{(3)}} & =710+552 x+118 x^{2}+5 x^{3} \\
A_{9, \tau^{(3)}} & =4168+3146 x+603 x^{2}+19 x^{3} \\
A_{10, \tau^{(3)}} & =29774+21666 x+5370 x^{2}+2697 x^{3}+14 x^{4} \\
A_{11, \tau^{(3)}} & =149030+152170 x+27000 x 2+25536 x^{3}+56 x^{4}
\end{aligned}
$$

Here is the list of the first few values of $\operatorname{GMP}_{2 n, \tau^{(5)}}(x)$.

$$
\begin{aligned}
& \operatorname{GMP}_{1, \tau^{(5)}}(x)=1 \\
& \operatorname{GMP}_{2, \tau^{(5)}}(x)=1 \\
& \operatorname{GMP}_{3, \tau^{(5)}}(x)=-1 \\
& \operatorname{GMP}_{4, \tau^{(5)}}(x)=-2+x \\
& \operatorname{GMP}_{5, \tau^{(5)}}(x)=4-3 x \\
& \operatorname{GMP}_{6, \tau^{(5)}}(x)=14-14 x+x^{2} \\
& \operatorname{GMP}_{7, \tau^{(5)}}(x)=-39+44 x-6 x^{2} \\
& \operatorname{GMP}_{8, \tau^{(5)}}(x)=-168+252 x-86 x^{2}+x^{3} \\
& G M P_{9, \tau^{(5)}}(x)=594-1002+416 x^{2}-7 x^{3} \\
& G M P_{10, \tau^{(5)}}(x)=3352-6704 x+3782 x^{2}-430 x^{3}+x^{4} \\
& G M P_{11, \tau^{(5)}}(x)=-13814+30264 x-19404 x^{2}+2962 x^{3}-9 x^{4}
\end{aligned}
$$




$$
G M P_{12, \tau^{(5)}}(x)=-91038+224751 x-180196 x^{2}+48387 x^{3}-1906 x^{4}+x^{5}
$$

Plugging these values into the generating functions (6) and (7), we have computed the following table of values of $A_{n, \tau^{(5)}}(x)=\sum_{\sigma \in \mathcal{A}_{n}} x^{\tau^{(5)}-\operatorname{mch}(\sigma)}$.

$$
\begin{aligned}
& A_{1, \tau^{(5)}}=1 \\
& A_{2, \tau^{(5)}}=1 \\
& A_{3, \tau^{(5)}}=2 \\
& A_{4, \tau^{(5)}}=4+x \\
& A_{5, \tau^{(5)}}=14+2 x \\
& A_{6, \tau^{(5)}}=44+16 x+x^{2} \\
& A_{7, \tau^{(5)}}=214-56 x+2 x^{2} \\
& A_{8, \tau^{(5)}}=896+448 x+40 x^{2}+x^{3} \\
& A_{9, \tau^{(5)}}=5610+2190 x+134 x^{2}+2 x^{3} \\
& A_{10, \tau^{(5)}}=29392+18496 x+2552 x^{2}+80 x^{3}+x^{4} \\
& A_{11, \tau^{(5)}}=224878+116776 x+11880 x^{2}+256 x^{3}+2 x^{4}
\end{aligned}
$$

\section{Double rise pairs and double descent pairs}

Suppose that $\sigma=\sigma_{1} \ldots \sigma_{n} \in \mathcal{A}_{n}$. Then we say that $(2 i-1)(2 i)$ is a double rise (double descent) pair in $\sigma$ if both $\sigma_{2 i-1}<\sigma_{2 i+1}$ and $\sigma_{2 i}<\sigma_{2 i+2}\left(\sigma_{2 i-1}>\sigma_{2 i+1}\right.$ and $\left.\sigma_{2 i}>\sigma_{2 i+2}\right)$. It is easy to see that $(2 i-1)(2 i)$ is a double rise pair if and only if $\operatorname{red}\left(\sigma_{2 i-1} \sigma_{2 i} \sigma_{2 i+1} \sigma_{2 i+2}\right)=$ 1324 so that the number of double rise pairs in $\sigma$ is just the number of 1324-matches in $\sigma$. Similarly, $(2 i-1)(2 i)$ is a double descent pair if and only if $\operatorname{red}\left(\sigma_{2 i-1} \sigma_{2 i} \sigma_{2 i+1} \sigma_{2 i+2}\right) \in$ $\{3412,2413\}$ so that if $D=\{3412,2413\}$, then the number of double descent pairs in $\sigma$ is just the number of $D$-matches in $\sigma$.

In general, if $\Upsilon \subseteq \mathcal{A}_{4}$, we say that $\sigma \in \mathcal{A}_{2 n}$ is a maximum packing for $\Upsilon$ if $\Upsilon$-mch $(\sigma)=$ $n-1$. We say that $\sigma \in S_{2 n}$ is a generalized maximum packing for $\Upsilon$ if we can break $\sigma$ into consecutive blocks $\sigma=B_{1} \ldots B_{k}$ such that

1. for all $1 \leqslant j \leqslant k, B_{j}$ is either an increasing sequence of length 2 or $\operatorname{red}\left(B_{j}\right)$ is a maximum packing for $\Upsilon$ of length $2 s$ for some $s \geqslant 2$ and

2. for all $1 \leqslant j \leqslant k-1$, the last element of $B_{j}$ is less than the first element of $B_{j+1}$.

Similarly, we say that $\sigma \in \mathcal{A}_{2 n+1}$ is a maximum packing for $\Upsilon$ if $\Upsilon$-mch $(\sigma)=n-1$. We say that $\sigma \in S_{2 n+1}$ is a generalized maximum packing for $\Upsilon$ if we can break $\sigma$ into consecutive blocks $\sigma=B_{1} \ldots B_{k}$ such that

1. for all $1 \leqslant j<k, B_{j}$ is either an increasing sequence of length 2 or $\operatorname{red}\left(B_{j}\right)$ is a maximum packing for $\Upsilon$ of length $2 s$ for some $s \geqslant 2$,

2. $B_{k}$ is block of length 1 , and 
3. for all $1 \leqslant j \leqslant k-1$, the last element of $B_{j}$ is less than the first element of $B_{j+1}$.

Then we can define the generalized maximum packing polynomials $G M P_{2 n, \Upsilon}(x)$ and $G M P_{2 n+1, \Upsilon}(x)$ in the same manner that we defined $G M P_{2 n, \tau^{(i)}}(x)$ and $G M P_{2 n+1, \tau^{(i)}}(x)$.

If $\Upsilon \subseteq \mathcal{A}_{4}$, the proof of Theorem 1 goes through without change if we replace maximum packings for $\tau$ with maximum packings for $\Upsilon$ and generalized maximum packings for $\tau$ by generalized maximum packings for $\Upsilon$ throughout the proof. Thus we have the following theorem.

Theorem 9. Let $\Upsilon \subseteq \mathcal{A}_{4}$. Then

$$
\begin{aligned}
A_{\Upsilon}(t, x) & :=1+\sum_{n \geqslant 1} \frac{t^{2 n}}{(2 n) !} \sum_{\sigma \in \mathcal{A}_{2 n}} x^{\Upsilon-\operatorname{mch}(\sigma)} \\
& =\frac{1}{1-\sum_{n \geqslant 1} G M P_{2 n, \Upsilon}(x) \frac{t^{2 n}}{(2 n) !}}
\end{aligned}
$$

and

$$
\begin{aligned}
B_{\Upsilon}(t, x) & :=\sum_{n \geqslant 1} \frac{t^{2 n-1}}{(2 n-1) !} \sum_{\sigma \in \mathcal{A}_{2 n-1}} x^{\Upsilon-\operatorname{mch}(\sigma)} \\
& =\frac{\sum_{n \geqslant 1} G M P_{2 n-1, \Upsilon}(x) \frac{t^{2 n-1}}{(2 n-1) !}}{1-\sum_{n \geqslant 1} G M P_{2 n, \Upsilon}(x) \frac{t^{2 n}}{(2 n) !}}
\end{aligned}
$$

If we can compute $G M P_{n, D}(x)$, we would have the generating function for the distribution of double descents in $\mathcal{A}_{n}$. We can compute $\operatorname{mp}_{2 n, D}$ and $\operatorname{mp}_{2 n+1, D}$. That is, we have the following theorem.

Theorem 10. For all $n \geqslant 1, m p_{2 n, D}=C_{n}$ and $m p_{2 n+1, D}=C_{n+1}$.

Proof. It is easy to see that $\operatorname{mp}_{2 n, D}$ equals the number of $F \in \mathcal{F}_{2, n}$ such that for each $i<n$, there is either a $P^{(2)}$-match or a $P^{(5)}$-match starting in column $i$. Let $F^{r}$ be the reverse of $F$. That is, the first row of $F^{r}$ is $F(1, n), F(1, n-1) \ldots, F(1,1)$ and the second row of $F^{r}$ is $F(2, n), F(2, n-1) \ldots, F(2,1)$, reading from left to right. For example,

$$
\left(P^{(3)}\right)^{r}=P^{(1)}=\begin{array}{|l|l|}
\hline 3 & 4 \\
\hline 1 & 2 \\
\hline
\end{array} \text { and }\left(P^{(5)}\right)^{r}=\begin{array}{|l|l|}
\hline 2 & 4 \\
\hline 1 & 3 \\
\hline
\end{array}
$$

It is easy to see that $F \in \mathcal{F}_{2, n}$ has the property that for each $i<n$, there is either a $P^{(2)}$-match or a $P^{(5)}$-match starting at column $i$ if and only if $F^{r} \in \mathcal{F}_{2, n}$ has the property that for each $i<n$, there is either a $\left(P^{(2)}\right)^{r}$-match or a $\left(P^{(5)}\right)^{r}$-match starting at column $i$. But note that $\left(P^{(3)}\right)^{r}$ and $\left(P^{(5)}\right)^{r}$ are the two standard tableaux of shape $(2,2)$. Thus $F^{r}$ has the property that for each $i<n$, there is either a $\left(P^{(2)}\right)^{r}$-match or a $\left(P^{(5)}\right)^{r}$-match starting at column $i$ if and only if $F^{r}$ is a standard tableau of shape $(n, n)$. But it follows from the Frame-Robinson-Thrall hook formula [10] for the number of standard tableaux 
of a given shape $\lambda$ that the number of standard tableaux of shape $(n, n)$ is the Catalan number $C_{n}$. Thus $\operatorname{mp}_{2 n, D}=C_{n}$.

The graph $G_{D}$ associated with $D$ is pictured on the right in the first line of Figure 15. Then we can construct the graphs $G_{D, n}$ and $G_{D, n}^{+}$using $G_{D}$ in the same way that we constructed the graphs $G_{n, P^{(i)}}$ and $G_{n, P^{(i)}}^{+}$from $G_{P^{(i)}}$. For example, the graphs $G_{D, 6}$ and $G_{D, 6}^{+}$are pictured on line 2 of Figure 15. Then $\mathrm{mp}_{2 n, D}$ is the number of linear extensions of the poset determined by $G_{n, D}$ and $\mathrm{mp}_{2 n+1, D}$ is the number of linear extensions of the poset determined by $G_{n, D}^{+}$.

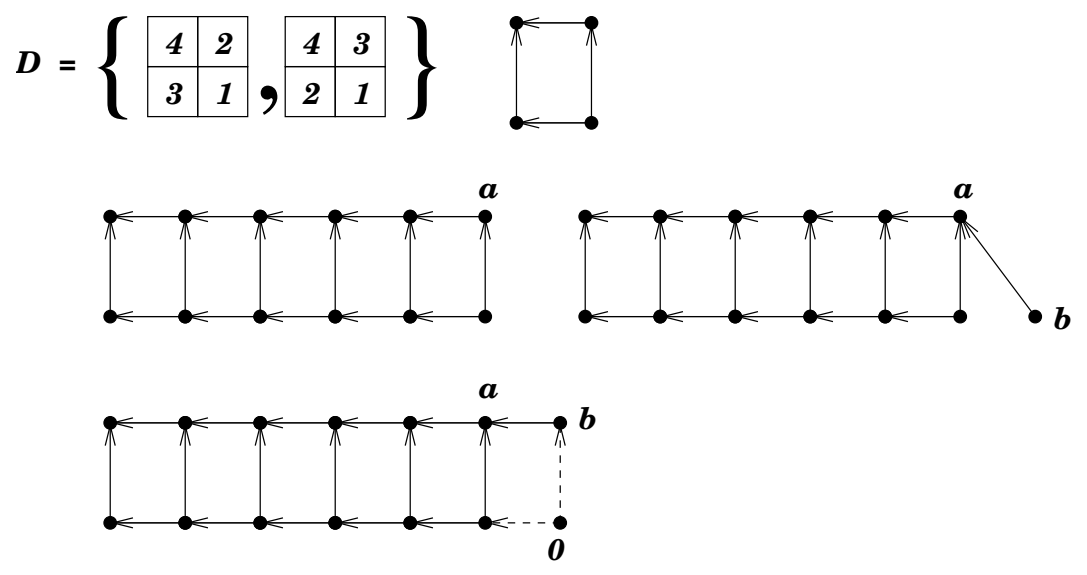

Figure 15: The graphs $G_{n, D}$ and $G_{n, D}^{+}$.

We claim that the number of linear extensions of the poset determined by $G_{n, D}^{+}$is just $C_{n+1}$. Note that in $G_{n, D}$, the element in the bottom right-hand corner must be the first element in any linear extension of the poset determined by $G_{n, D}$. Now create a new graph $G_{n, D}^{++}$by adding a new element 0 and new directed edges connecting 0 to the element in the bottom right hand corner of $G_{n, D}^{+}$and 0 to $b$ to form a graph $G_{n, D}^{++}$. It is easy to see that the number of linear extensions of the poset determined by $G_{n, D}^{+}$is equal to the number of linear extensions of the poset determined by $G_{n, D}^{++}$. However the number of linear extensions of the poset determined by $G_{n, D}^{++}$is just the number of linear extensions of the poset determined by $G_{n+1, D}$ which is $C_{n+1}$.

Unfortunately elements of $\mathcal{M P}_{2 n, D}$ do not end in 1 or $2 n$ so that there does not seem to be any way to develop simple recursions for $G M P_{2 n, D}(x)$ or $G M P_{2 n+1, D}(x)$. Nevertheless, J. Harmse [12] computed the following initial values of $G M P_{n, D}(x)$

$$
\begin{aligned}
& G M P_{1, D}(x)=1 \\
& G M P_{2, D}(x)=1 \\
& G M P_{3, D}(x)=-1 \\
& G M P_{4, D}(x)=2 x-3 \\
& G M P_{5, D}(x)=6-5 x
\end{aligned}
$$




$$
\begin{aligned}
G M P_{6, D}(x) & =24-28 x+5 x^{2} \\
G M P_{7, D}(x) & =-64+84 x-21 x^{2} \\
G M P_{8, D}(x) & =-369+648 x-294 x^{2}+14 x^{3} \\
G M P_{9, D}(x) & =1288-2439 x+1236 x^{2}-84 x^{3} \\
G M P_{10, D}(x) & =8970-20792 x+15189 x^{2}-3408 x^{3}+42 x^{4} \\
G M P_{11, D}(x) & =-31121+73723 x-54978 x^{2}+12705 x^{3}-330 x^{4} \\
G M P_{12, D}(x) & =-323736+933223 x-937838 x^{2}+369138 x^{3}-40920 x^{4}+132 x^{5}
\end{aligned}
$$

Plugging these values into the generating functions (42) and (42), we have computed the following table of values of $A_{n, D}(x)=\sum_{\sigma \in \mathcal{A}_{n}} x^{D-\operatorname{mch}(\sigma)}$.

$$
\begin{aligned}
A_{1, D} & =1 \\
A_{2, D} & =1 \\
A_{3, D} & =2 \\
A_{4, D} & =3+2 x \\
A_{5, D} & =11+5 x \\
A_{6, D} & =24+32 x+5 x^{2} \\
A_{7, D} & =125+133 x+14 x^{2} \\
A_{8, D} & =345+760 x+266 x^{2}+14 x^{3} \\
A_{9, D} & =1341+4359 x+1194 x^{2}+42 x^{3} \\
A_{10, D} & =7890+24928 x+15609 x^{2}+2052 x^{3}+42 x^{4} \\
A_{11, D} & =17752+162570 x+115401 x^{2}+2937 x^{3}+132 x^{4}
\end{aligned}
$$

A. Duane, in his Ph.D. thesis [7], showed that the techniques that we have developed in this paper can be extended to find generating functions for the distribution of the number of $\tau$-matches in $\mathcal{A}_{n}$ where $\tau \in \mathcal{A}_{2 j}$ is an up-down minimal overlapping permutation. Here $\tau \in \mathcal{A}_{2 j}$ is said to be an up-down minimal overlapping permutation if the smallest $i$ such that there exists a $\sigma \in \mathcal{A}_{2 i}$ such that $\tau$-mch $(\sigma)=2$ is $4 j-2$. Also the techniques that we have developed in this paper can be generalized to find the generating functions for the distribution of the number of consecutive matches in generalized $k$-Euler permutations. That is, let $E_{n}^{(k)}=\left\{\sigma \in S_{n}: \operatorname{Des}(\sigma)=\{k j: j \geqslant 1\} \cap[n-1]\right\}$. In particular, we can generalize the results of this paper to study the distribution of $\tau$-matches in $E_{n}^{(k)}$ where $\tau \in E_{2 k}^{(k)}$.

\section{Acknowledgements}

We wish to thank the anonomous referee for many suggested improvements in the presentation of this paper 


\section{References}

[1] D. Beck and J. Remmel, Permutation enumeration of the symmetric group and the combinatorics of symmetric functions, J. of Combinatorial Theory (A), 72 (1995), $1-49$.

[2] D. Beck, J. Remmel, and T. Whitehead, The combinatorics of transition matrices between the bases of the symmetric functions and the $B_{n}$ analogues, Discrete Math., 153 (1996), 3-27.

[3] F. Brenti, Permutation enumeration, symmetric functions, and unimodality, Pacific J. Math., 157 (1993), 1-28.

[4] F. Brenti, A class of $q$-symmetric functions arising from plethysm, J. Combinatorial Theory (A), 91 (2000), 137-170.

[5] L. Carlitz, Enumeration of up-down permutations by the number of rises, Pacific J. Math., 45 (1973), 49-59.

[6] L. Carlitz and R. Scoville, Enumeration of up-down permutations by upper records, Monatsh. Math., 79 (1975), 3-12.

[7] A. S. Duane, Pattern matching statistics in the permutations $S_{n}$ and the alternating permutations $A_{n}$ for minimally overlapping patterns, Ph.D. Thesis, University of California, San Diego, (2013).

[8] A.S. Duane and J.B. Remmel, Minimal overlapping patterns in colored permutations, Electronic J. Combinatorics, 18(2) (2011), P25, 34 pgs.

[9] Ö. Eg̃eciog̃lu and J. Remmel, Brick tabloids and connection matrices between bases of symmetric functions, Discrete Applied Math. 34 (1991), 107-120.

[10] J.S. Frame, G. De B. Robinson, and R.M. Thrall, The hook graphs of the symmetric group, Canad. J. Math., 6 (1954), 316-324.

[11] I.P. Goulden and D.M. Jackson, Combinatorial Enumeration, A Wiley-Interscience Series in Discrete Mathematics, John Wiley \& Sons Inc, New York, (1983).

[12] J. Harmse, private communication (2012).

[13] J. Harmse and J.B. Remmel, Patterns in column strict fillings of rectangular arrays, Pure Math. and Applications, 22 (2011), 131-171.

[14] G. Hong, Catalan numbers in pattern-avoiding permutations, MIT Undergraduate J. Mathematics, 10 (2008), 53-68.

[15] S. Kitaev, A. Niedermaier, J.B. Remmel, and A. Riehl, Generalized pattern matching conditions for $C_{k} 2 S_{n}$, ISRN Combinatorics, 2013, Article ID634823 (2013), 21. pgs.

[16] T.M. Langley, Alternative transition matrices for Brenti's q-symmetric functions and a class of $q, t$-symmetric functions on the hyperoctahedral group, Proceedings of the 2002 Conference on Formal Power Series and Algebraic Combinatorics, Melbourne Australia. 
[17] T.M. Langley and J.B. Remmel, Enumeration of $m$-tuples of permuations and a new class of power bases for the space of symmetric functions, Advances in App. Math., 36 (2006), 30-66.

[18] J.B. Lewis, Pattern avoidance in alternating permutations and tableaux, DMTCS Proceedings, 22nd International Conference on Formal Power Series and Algebraic Combinatorics (FPSAC 2010), (2010), 391-402.

[19] T. Mansour, Restricted 132-alternating permutations and Chebyshev polynomials, Annals of Combinatorics, 7 (2003), 201-227.

[20] T. Mansour and A. Robertson, Refined restricted permutations avoiding subsets of patterns of length 3, Annals of Combinatorics, 6 (2002), 407-418.

[21] A. Mendes, Building generating functions brick by brick, Ph.D. thesis, University of California, San Diego, (2004).

[22] A. Mendes and J.B. Remmel, Permutations and words counted by consecutive patterns, Advances Appl. Math., 37(4), (2006), 443-480.

[23] A. Mendes and J. Remmel, Generating functions for statistics on $C_{k} 2 S_{n}$, Sém. Lothar. Comb. 54A: Article B54At, (2005/07).

[24] A. Mendes and J.B. Remmel, Generating Functions from Symmetric Functions, preprint.

[25] A. Mendes, J.B. Remmel, and A. Riehl, Permutations with $k$-regular descent patterns, Permutation Patterns, (S. Linton, N. Ruškuc, V. Vatter, eds.), London. Math. Soc. Lecture Notes 376, (2010), 259-286.

[26] D. Ram, J.B. Remmel, and T. Whitehead, Combinatorics of the $q$-basis of symmetric functions, J. Comb. Th. Series A, 76(2) (1996), 231-271.

[27] J. D. Wagner, The permutation enumeration of wreath products and cyclic and symmetric groups, Advances in Appl. Math., 30 (2003), 343-368.

[28] R.P. Stanley, Catalan addendum to Enumerative Combinatorics, 2013. Available online at http://www-math.mit.edu/ rstan/ec/catadd.pdf 